\title{
RANDOMLY PERTURBED ERGODIC AVERAGES
}

\author{
JAEYONG CHOI AND KARIN REINHOLD-LARSSON
}

(Communicated by Nimish Shah)

\begin{abstract}
We consider a class of random ergodic averages, containing averages along random non-integer sequences. For such averages, Cohen \& Cuny obtained uniform universal pointwise convergence for functions in $L^{2}$ with $\int \max (1, \log (1+|t|)) d \mu_{f}<\infty$ via a uniform estimation of trigonometric polynomials. We extend this result to $L^{2}$ functions satisfying the weaker condition $\int \max (1, \log \log (1+|t|)) d \mu_{f}<\infty$. We also prove that uniform universal pointwise convergence in $L^{2}$ holds for the corresponding smoothed random averages or for random averages whose kernels exhibit sufficient decay at infinity.
\end{abstract}

\section{INTRODUCTION}

In this work, we adopt a general framework for describing random averaging operators. $(\Omega, \mathcal{F}, P)$ denotes a probability space, and $\left\{\nu_{k}\right\}_{k \in \mathbb{N}^{r}}$ a sequence of independent transition measures on $\Omega \times \mathbb{R}^{d}, \nu_{k}: \Omega \times \mathbb{R}^{d} \mapsto \mathbb{C}^{d}$. See Section 2 for a precise definition of independent transition measures. $(X, \mathcal{D}, m)$ denotes a probability space and $\left\{T_{t}\right\}_{t \in\left(\mathbb{R}^{+}\right) d}$ a continuous semi-flow of positive isometries acting on $L^{2}(X)$. Each measure $\nu_{k}(\omega,$.$) on \left(\mathbb{R}^{+}\right)^{d}$ defines a bounded operator in $L^{2}(X)$ as a weighted average over the flow:

$$
\nu_{k}^{\omega} f(x)=\int_{\mathbb{R}^{d}} T_{t} f(x) \nu_{k}(\omega, d t) .
$$

We denote their averages by

$$
K_{n}^{\omega} f(x)=\frac{1}{b_{n}} \sum_{k \in[1, n]^{r}} \nu_{k}^{\omega}(f)(x), \text { where } b_{n}=\sum_{k \in[1, n]^{r}} E\left(\left|\nu_{k}\left(., \mathbb{R}^{d}\right)\right|\right) .
$$

This approach includes averages of the form

$$
\frac{1}{n^{r}} \sum_{k \in[1, n]^{r}} T_{X_{k}(\omega)} f(x) \text {, or } \frac{1}{n^{r}} \sum_{k \in[1, n]^{r}} \frac{1}{\left(2 \epsilon_{k}(\omega)\right)^{d}} \int_{\|t\|<\epsilon_{k}(\omega)} T_{X_{k}(\omega)+t} f(x) d t,
$$

where $X_{k}: \Omega \mapsto\left(\mathbb{R}^{+}\right)^{d}$ and $\epsilon_{k}: \Omega \mapsto \mathbb{R}^{+}$are sequences of random variables; and averages of the form

$$
\frac{1}{b_{n}} \sum_{k \in[1, n]^{r}} Y_{k}(\omega) T_{n_{k}} f(x),
$$

where $Y_{k}: \Omega \mapsto \mathbb{C}$ is a sequence of random variables with $b_{n}=\sum_{k \in[1, n]^{r}} E\left(\left|Y_{k}\right|\right)$.

Received by the editors October 10, 2018, and, in revised form, August 31, 2020, and September $11,2020$.

2020 Mathematics Subject Classification. Primary 37A05, 28D05.

Key words and phrases. Random ergodic averages, universal convergence.

(C) 2021 by the authors under Creative Commons Attribution-Noncommercial 3.0 License (CC BY NC 3.0) 
Before addressing the case of random averages, we review some key results for ergodic averages along subsequences. A sequence of bounded operators $\left\{T_{n}\right\}_{n \in \mathbb{N}}$ on $L^{2}(X)$ has the strong sweeping out property if, for any $\epsilon>0$, there exists a set $E$ with $0<m(E)<\epsilon$, such that $\limsup _{n \rightarrow \infty} T_{n} 1_{E}=1$ a.e. and $\lim _{\inf } \operatorname{sum}_{n \rightarrow \infty} T_{n} 1_{E}=0$ a.e..

Let $(X, \mathcal{D}, m)$ be a non-atomic probability space and $\left\{\tau_{t}\right\}_{t \in \mathbb{R}}$ an aperiodic, ergodic, measure preserving flow on it. Ackoglu, Bellow, del Junco and Jones [1] showed that, for any increasing sequence of integers $\left\{n_{k}\right\}_{k \in \mathbb{N}}$, if $\left\{t_{k}\right\}_{k \in \mathbb{N}}$ is a sequence such that $t_{k} \mapsto 0$, the averages $B_{n} f(x)=\frac{1}{n} \sum_{k=1}^{n} f\left(\tau_{n_{k}+t_{k}} x\right)$ have the strong sweeping out property and, therefore, there exist bounded functions for which pointwise convergence fails on sets of positive measure. Bergelson, Boshernitzan and Bourgain [7] had used Bourgain's entropy method [1] to show that the averages $B_{n} f$ diverge a.e. for some $f \in L^{\infty}$ when the sequence $\left\{t_{k}\right\}$ is independent over the rationals. Ackoglu, del Junco and Lee 2] proved that the related averages, $\frac{1}{n} \sum_{k=1}^{n} f\left(\left\{x+t_{k}\right\}\right)$, for $f$ in $[0,1)$, have the $\delta$-sweeping out property. In [3], Ackoglu et al. showed that these averages have, in fact, the strong sweeping out property. When the derministic sequence $\left\{t_{k}\right\}_{k \in \mathbb{N}}$ is replaced by a random sequence, uniform positive results are obtained.

Definition 1.1. A sequence of operators $\left\{R_{n}\right\}_{n \in \mathbb{N}}$ of the form

$$
R_{n} f(x)=\int_{\mathbb{R}^{d}} T_{t} f(x) \mu_{n}(d t),
$$

where $\left\{\mu_{n}\right\}_{n \in \mathbb{N}}$ is a sequence of finite measures on $\left(\mathbb{R}^{+}\right)^{d}$, is universally good in $S \subset L^{2}$, if for any probability space $(X, \mathcal{D}, m)$ and any $\left\{T_{t}\right\}_{t \in\left(\mathbb{R}^{+}\right)^{d}}$, a continuous semi-flow of positive isometries acting on $L^{2}(X), \lim _{n \rightarrow \infty} R_{n} f(x)$ exists a.e. for any $f \in S(X)$. When $R_{n} f(x)=\frac{1}{n^{r}} \sum_{k \in[1, n]^{r}} T_{n_{k}} f(x)$, we simply say the sequence $\left\{n_{k}\right\}_{k \in \mathbb{N}^{r}} \subset\left(R^{+}\right)^{d}$ is universally good in $S$.

Definition 1.2. With the notation from (1.0.1), we say the averaging operators $\left\{K_{n}\right\}_{n \in \mathbb{N}}$ are uniformly universally good in $S \subset L^{2}$ if there exists $\Omega^{\prime} \subset \Omega$, with $P\left(\Omega^{\prime}\right)=1$ such that, for all $\omega \in \Omega^{\prime},\left\{K_{n}^{\omega}\right\}_{k \in \mathbb{N}}$ is universally good in $S$. When $\nu_{k}(\omega, x)=\delta_{r_{k}(\omega)}(x)$, with $r_{k}: \Omega \mapsto\left(\mathbb{R}^{+}\right)^{d}$ a sequence of random vectors; we simply say that the sequence $\left\{r_{k}\right\}$ is uniformly universally good in $S$.

In what follows, $\left\{n_{k}\right\}_{k \in \mathbb{N}}$ is a non-decreasing sequence in $\mathbb{R}^{+},\left\{\gamma_{k}\right\}_{k \in \mathbb{N}}$ and $\left\{\epsilon_{k}\right\}_{k \in \mathbb{N}}$ are independent sequences of random variables defined on a probability space $(\Omega, \mathcal{F}, P)$ and $\left\{T_{t}\right\}_{t \in\left(\mathbb{R}^{+}\right)} \subset \mathbb{R}^{+}$, a semi-flow of positive isometries.

Reinhold [32] showed the averages $\frac{1}{n} \sum_{k=1}^{n} \frac{1}{2\left|\epsilon_{k}(\omega)\right|} \int_{|t|<\left|\epsilon_{k}(\omega)\right|} T_{k+\gamma_{k}(\omega)+t} f(x) d t$, are uniformly universally good in $L^{p}, p \geq 1$, provided $e_{k}^{-1} \in L^{q}, \frac{1}{p}+\frac{1}{q}$. Schneider [38] showed that the averages $G_{n} f(\omega, x)=\frac{1}{n} \sum_{k=1}^{n} T_{n_{k}+\gamma_{k}(\omega)} f(x)$, are uniformly universally good in $L^{2}$ for the sequence $n_{k}=k^{2}$ and $\gamma_{k}$ i.i.d. random variables taking values 1 and -1 with probability $1 / 2$. Schneider [39] extended this result for universally good in $L^{2}$ integer-valued sequences with the growth condition $n_{k}=O\left(2^{k^{s}}\right)$, for some $s \in(0,1)$, and where $\left\{\gamma_{k}\right\}_{k \in \mathbb{N}}$ are integer-valued independent random variables. Duran and Schneider [22] applied the techniques to averages of the form $\frac{1}{n} \sum_{k=1}^{n} T_{X_{k}(\omega)} f(x)$ where the distribution of the integer valued independent random variables $\left\{X_{k}\right\}_{k \in \mathbb{N}}$ is generated by the convolution of a given law, $d \mathbb{P}_{X_{k}}=d \mathbb{P}_{Y}^{*\left(S_{k}\right)}$, for all $k \geq 1,(Y$ an integrable random variable). They also showed 
that the averages $\frac{1}{n} \sum_{k=1}^{n} X_{k}(\omega) T_{n_{k}} f(x)$ are uniformly universally good in $L^{2}$ when $n_{k}=O\left(2^{k^{s}}\right)$ is a sequence of integers, without requiring it to be universally good in $L^{2}$, and $\left\{X_{k}\right\}_{k \in \mathbb{N}}$ are centered i.i.d. with finite variance.

Cohen and Cuny [15] obtained uniformly universally good results for the averages $G_{n} f(\omega, x)$ for sequences $\left\{\gamma_{k}\right\}_{k \in \mathbb{N}}$ that do not take integer values. In light of 3 , 7], pointwise convergence of $G_{n} f(\omega, x)$ fails for some functions in $L^{2}$ but positive results are obtained by considering a subclass. Cohen and Cuny 15 proved, under certain condition on the sequences $\left\{n_{k}\right\}_{k \in \mathbb{N}}$ and $\left\{\gamma_{k}\right\}_{k \in \mathbb{N}}$, the averages $\left\{G_{n} f\right\}_{k \in \mathbb{N}}$ are uniformly universally good for $f \in L^{2}$ such that $\int \log (2+|t|) d \mu_{f}(t)<\infty$. Their approach applies to a wider class of random averages as wells as to the study of convergence of certain random series.

Definition 1.3. Let $(\Omega, \mathcal{F}, P)$ be a probability space. We say the operators defined by the sequence of transition measures $\left\{\nu_{k}\right\}_{k \in \mathbb{N}^{r}}$ are uniformly norm summable in $S \subset L^{2}$ if there exists a set $\Omega^{\prime} \subset \Omega$ with $P\left(\Omega^{\prime}\right)=1$, such that, for every $\omega \in \Omega^{\prime}$, for any probability space $(X, \mathcal{D}, m)$, any continuous semi-flow of positive isometries $\left\{T_{t}\right\}_{t \in\left(\mathbb{R}^{+}\right)^{d}}$ in $L^{2}(X)$, and any $f \in S(X)$,

$$
\left\|\sum_{k \in \mathbb{N}^{r}} \frac{\nu_{k}^{\omega} f-E\left(\nu_{k}\right) f}{|k|^{r}}\right\|_{2} \leq C\|f\|_{S},
$$

where $|k|=\max _{1 \leq i \leq d}\left|k_{i}\right|$. When $r_{k}: \Omega \mapsto\left(\mathbb{R}^{+}\right)^{d}$ is a sequence of random variables and $\nu_{k}(\omega, x)=\delta_{r_{k}(\omega)}(x)$, we say that the sequence $\left\{r_{k}\right\}$ is uniformly normsummable.

Define $(\log \psi(t))^{+}=\log _{2} \psi(t)$ if $\psi(t)>2$, and 1 otherwise.

Definition 1.4. $(\log \psi)^{+} L^{2}=\left\{f \in L^{2}: \int(\log \psi|t|)^{+} d \mu_{f}<\infty\right\}$. For convenience, we'll write $\|f\|_{(\log \psi)^{+} L^{2}}=\left(\int(\log \psi|t|)^{+} d \mu_{f}\right)^{1 / 2}$.

Theorem 1.5 (Cohen and Cuny, [15, Theorem 4.12]). Let $\left\{X_{n}\right\}_{n \in \mathbb{N}} \subset\left(\mathbb{R}^{+}\right)^{d}$ be i.i.d random variables with $E\left(\left|X_{1}\right|^{\alpha}\right)<\infty$ for some $\alpha>0$. Let $\left\{n_{k}\right\}_{k \in \mathbb{N}} \subset\left(\mathbb{R}^{+}\right)^{d}$, with $\left|n_{m}\right|^{*}=\max _{k \leq m}\left|n_{k}\right|=O\left(2^{m^{\beta}}\right)$, for some $0<\beta<1$. Then, $\left\{n_{k}+X_{k}\right\}_{k \in \mathbb{N}}$ is uniformly norm summable in $(\mathrm{log})^{+} L^{2}$. In particular, centered averages along the sequence $\left\{n_{k}+X_{k}\right\}$

$$
\frac{1}{n} \sum_{k=1}^{n}\left(T_{n_{k}+X_{k}} f-E_{\Omega} T_{n_{k}+X_{k}} f\right)
$$

are uniformly universally good in $(\log )^{+} L^{2}$.

In section 2, we present Cohen's 14 extension of Cohen and Cuny's 15 uniform estimates for almost periodic polynomials corresponding to the case of FourierStieltjes transform of transition measures. Such estimates are essential for uniform universal results in $L^{2}$. In Section 3, we prove the boundedness for a square function for the centered averages $\left\{K_{n}^{\omega} f-E_{\Omega}\left(K_{n} f\right)\right\}_{n \in I(\rho)}$, where $I(\rho)=\left\{\left\lfloor\rho^{n}\right\rfloor, n \in \mathbb{N}\right\}$ $(\rho>1)$; an estimate that suffices for a uniform universal result under the weaker condition $\int(\log \log (|t|))^{+} d \mu_{f}(t)<\infty$.

Definition 1.6. Given a set of complex numbers $\left\{x_{n}\right\}_{n \in I}$, where $I$ is a countable index set, define its $s$-variation norm as

$$
\left\|\left\{x_{n}\right\}_{n \in I}\right\|_{v(s)}=\sup \left(\sum_{j=1}^{\infty}\left|x_{n_{j}}-x_{n_{j+1}}\right|^{s}\right)^{1 / s}
$$


where the supremum is taken over all possible non-decreasing subsequences $\left\{n_{j}\right\}$ in $I$. When the index set is $\mathbb{N}$, we simply write $\left\|\left\{x_{n}\right\}_{n \in \mathbb{N}}\right\|_{v(s)}=\left\|x_{n}\right\|_{v(s)}$.

Note that when the sequence is given by a sequence of Lebesgue measurable complex-valued functions $\left\{f_{n}\right\}_{n \in \mathbb{N}}$, then its $s$-variation norm

$$
\left\|f_{n}(x)\right\|_{v(s)}=\sup _{\left\{\text {all non-decreasing }\left\{n_{j}\right\}\right\}}\left(\sum_{j=1}^{\infty}\left|f_{n_{j}}(x)-f_{n_{j+1}}(x)\right|^{s}\right)^{1 / s}
$$

is a Lebesgue measurable function. Variation norms are thus a useful tool for convergence, since the boundedness of the variation norm, $\left\|f_{n}(x)\right\|_{v(s)}<\infty$, implies convergence of $\left\{f_{n}(x)\right\}_{n}$.

Definition 1.7. A sequence of operators $\left\{R_{n}\right\}_{n \in I}$ as in Definition 1.1 is variationally good in $S \subset L^{2}$, if, for some $s>2$, there exits a constant $c(s)>0$ such that, for any probability space $(X, \mathcal{D}, m)$ and any $\left\{T_{t}\right\}_{t \in \mathbb{R}^{d}}$ a continuous semi-flow of positive isometries acting on $L^{2}(X)$

$$
\|\|\left\{R_{n} f\right\}_{n \in I}\left\|_{v(s)}\right\|_{2} \leq c(s)\|f\|_{S} .
$$

We say $\left\{K_{n}\right\}_{n \in I}$ is uniformly variationally good in $S \subset L^{2}$ if there exists $\Omega^{\prime} \subset \Omega$, with $P\left(\Omega^{\prime}\right)=1$ such that, for all $\omega \in \Omega^{\prime},\left\{K_{n}^{\omega}\right\}_{n \in I}$ is variationally good in $S$.

As before, when $R_{n}$ or $K_{n}$ are defined by a sequence (deterministic or random) we say that the sequence has the corresponding property.

Theorem 1.8. Let $\left\{\gamma_{n}\right\}_{n \in \mathbb{N}} \subset\left(\mathbb{R}^{+}\right)^{d}$ be independent random vectors such that, for some $0<\beta<1, \sum_{k \geq 1} P\left(\left|\gamma_{k}\right|>2^{k^{\beta}}\right)<\infty$. Let $\left\{n_{k}\right\}_{k \in \mathbb{N}} \subset\left(\mathbb{R}^{+}\right)^{d}$, and $\left|n_{k}\right|=O\left(2^{k^{\beta}}\right)$. Then the sequence

$$
\left\{\frac{1}{n} \sum_{k=1}^{n}\left(T_{n_{k}+\gamma_{k}} f-E_{\Omega} T_{n_{k}+\gamma_{k}} f\right)\right\}_{n \in I(\rho)}
$$

is uniformly universally good in $(\log \log )^{+} L^{2}$ for any $\rho>1$. Moreover, when $\left\{\gamma_{n}\right\}$ are i.i.d,

(a) if the sequence $\left\{n_{k}\right\}_{k \in \mathbb{N}}$ is universally good in $L^{2}$, then the sequence $\left\{n_{k}+\right.$ $\left.\gamma_{k}\right\}_{k \in \mathbb{N}}$ is uniformly universally good in $(\log \log )^{+} L^{2}$; and

(b) if in addition the sequence $\left\{n_{k}\right\}_{k \in I(\rho)}$ is variationally good in $L^{2}$ for $\rho>1$, then the the sequence $\left\{n_{k}+\gamma_{k}\right\}_{k \in I(\rho)}$ is uniformly variationally good in $(\log \log )^{+} L^{2}$.

In Section 3 we prove a general version of this theorem for averages $\left\{K_{n}\right\}$ of transition measures.

There is a large literature on sequences with various growth conditions which are universally good in $L^{2}$ and thus, this theorem gives conditions under which such sequences can be randomly perturbed with real valued sequences and still maintain uniform convergence on a subclass of $L^{2}$. Full variational inequalities along subsequences have been more difficult to obtain. The technique of handling variations to prove pointwise convergence was introduced in ergodic theory by Bourgain [10] for the regular ergodic averages. The methods were applied to sequences of primes and polynomial sequences [10]. Recently, Krause [27] showed the variational inequality for polynomial sequences and Zorin-Kranich 43 showed variational inequalities for weighted averages of powers of primes and for the polynomial sequence 
$\left(n, n^{2}, \ldots, n^{d}\right) \in \mathbb{Z}^{d}$. Therefore Theorem 1.8 yields uniform universal convergence results for the corresponding randomly perturbed averages.

Corollary 1.9. Let $\left\{\gamma_{n}\right\}_{n \in \mathbb{N}} \subset \mathbb{R}^{+}$be i.i.d. random variables such that, for some $0<\beta<1, \sum_{k \geq 1} P\left(\left|\gamma_{k}\right|>2^{k^{\beta}}\right)<\infty$. Let $p(x)$ be a polynomial with integer coefficients. Then the sequences $\left\{p(n)+\gamma_{n}\right\}_{n \in \mathbb{N}}$ and $\left\{\gamma_{n}\right\}_{n \in \mathbb{N}}$, are uniformly universally good in $(\log \log )^{+} L^{2}$.

In particular, if the $\left\{\gamma_{k}\right\}_{k \in \mathbb{N}}$ take value in $[0,1]$, the corresponding random Bellow's problem averages $\frac{1}{n} \sum_{k=1}^{n} T_{\gamma_{k}(\omega)} f(x)$, are uniformly universally and variationally good in $(\log \log )^{+} L^{2}$.

From Krause [27] and Zorin-Kranich [43] the next two applications follow.

Corollary 1.10. Let $\lambda(x)$ be the von Mangoldt function and let $\left\{\gamma_{n}\right\}_{n \in \mathbb{N}} \subset \mathbb{R}^{+}$ be i.i.d. random variables such that, for some $0<\beta<1, \sum_{k \geq 1} P\left(\left|\gamma_{k}\right|>2^{k^{\beta}}\right)<$ $\infty$. Then the averages $\frac{1}{N} \sum_{k=1}^{N} \lambda(k) T_{k+\gamma_{k}(\omega)} f(x)$ are uniformly universally and variationally good in $(\log \log )^{+} L^{2}$.

Corollary 1.11. Let $\left\{\gamma_{n}\right\}_{n \in \mathbb{N}^{d}} \subset\left(\mathbb{R}^{+}\right)^{d}$ be i.i.d. random vectors such that, for some $0<\beta<1, \sum_{k \geq 1} P\left(\left|\gamma_{k}\right|>2^{k^{\beta}}\right)<\infty$. Then the sequence $\left\{\left(n, n^{2}, \ldots, n^{d}\right)+\right.$ $\left.\gamma_{n}(\omega)\right\}$ is uniformly universally and variationally good in $(\log \log )^{+} L^{2}$.

In Section 4, full $L^{2}$ result are obtained when the kernels associated with the averaging operators have additional smoothing properties.

Let $\zeta: \mathbb{R}^{d} \rightarrow \mathbb{R}$ be positive, integrable with $\int_{\mathbb{R}^{d}} \zeta(t) d t=1$, and with support on $|t| \leq 1$. Let $L_{\epsilon} f(x)=\int_{\mathbb{R}^{d}} \zeta_{\epsilon}(t) T_{t} f(x) d t$ where $\zeta_{\epsilon}(t)=\frac{1}{\epsilon_{1} \cdots \epsilon_{d}} \zeta\left(\frac{t_{1}}{\epsilon_{1}}, \ldots, \frac{t_{d}}{\epsilon_{d}}\right)$. Let $\left\{\gamma_{k}\right\}_{k \in \mathbb{N}^{r}} \subset \mathbb{R}^{d}$ and $\left\{\epsilon_{k}\right\}_{k \in \mathbb{N}^{r}} \subset(0,1]^{d}$ be independent sequences of independent positive random vectors, and $\left\{n_{k}\right\}_{k \in \mathbb{N}^{r}} \subset\left(\mathbb{R}^{+}\right)^{d}$. From here on, $F_{n} f(x)$ denotes the following smoothed average around the observations $n_{k}+\gamma_{k}(\omega)$,

$$
F_{n} f(\omega, x)=F_{n} f(x)=\frac{1}{n^{r}} \sum_{k \in[1, n]^{r}} T_{n_{k}+\gamma_{k}(\omega)} L_{\epsilon_{k}(\omega)} f(x) .
$$

The regular averages along the sequence $\left\{n_{k}\right\}_{k \in \mathbb{N}^{r}}$ are denoted as

$$
A_{n} f(x)=\frac{1}{n^{r}} \sum_{k \in[1, n]^{r}} T_{n_{k}} f(x) .
$$

Theorem 1.12. Let $\left\{\gamma_{k}\right\}_{k \in \mathbb{N}^{r}} \subset \mathbb{R}^{d}$ and $\left\{\epsilon_{k}\right\}_{k \in \mathbb{N}^{r}} \subset(0,1]^{d}$ be two independent sequences of positive, independent random vectors, and $\left\{n_{k}\right\}_{k \in \mathbb{N}^{r}} \subset\left(\mathbb{R}^{+}\right)^{d}$. Assume they satisfy the following conditions:

(i) $E\left(\min _{1 \leq j \leq d}\left|\epsilon_{k, j}\right|^{-\alpha}\right)<\infty$ for some $\alpha>0$ (and any $k \in \mathbb{N}^{r}$ );

(ii) for some $0<\beta<1, \sum_{j \geq 1} j^{r-1} P\left(\left|\gamma_{j}\right|>2^{j^{r \beta}}\right)<\infty$;

(iii) $\left|n_{k}\right|=O\left(2^{|k|^{r \beta}}\right)$; and

(iv) $\sup _{t} \prod_{j=1}^{d} \max \left(1,\left|t_{j}\right|^{\alpha}\right)|\widehat{\zeta}(t)|<\infty$.

Then the sequence of transition probability measures $\left\{\delta_{n_{k}+\gamma_{k}} * \zeta_{\epsilon_{k}}\right\}_{k \in \mathbb{N}^{r}}$ is uniformly norm summable in $L^{2}$. Moreover, when $\left\{\epsilon_{k}\right\}$ and $\left\{\gamma_{k}\right\}$ are i.i.d., if the sequence $\left\{n_{k}\right\}$ is universally good in $L^{2}$, then the averaging operators $\left\{F_{n}\right\}_{n \in \mathbb{N}}$ are uniformly universally good in $L^{2}$; if in addition $\left\{A_{n} f\right\}$ are variationally good in $L^{2}$, then $\left\{F_{n}\right\}_{n \in \mathbb{N}}$ is uniformly variationally good in $L^{2}$. 
This theorem also holds for more general transition measures $\left\{\nu_{n}\right\}$ and their averages $\left\{K_{n}\right\}$, under equivalent requirements. See Theorem 4.2

Example 1.13. Let $d, r=1$. Let $\left\{\epsilon_{k}\right\}_{k \in \mathbb{N}} \subset \mathbb{N}$ be positive i.i.d with $E\left(1 / \epsilon_{1}\right)<\infty$, and let $\left\{\gamma_{k}\right\}_{k \in \mathbb{N}} \subset \mathbb{R}$ be bounded i.i.d. Let $\zeta=\chi_{[0,1]}$, then $\sup _{t} \max (1,|t|)|\widehat{\zeta}(t)| \leq 1$. From Krause [27, if $p(x)$ is a polynomial with integer coefficients, then the averages $F_{n}$ defined by the sequence of transition probability measures $\left\{\delta_{p(k)+\gamma_{k}} * \zeta_{\epsilon_{k}}\right\}_{k \in \mathbb{N}}$ or the smoothed-Bellow problem averages defined by $\left\{\delta_{\gamma_{k}} * \zeta_{\epsilon_{k}}\right\}_{k \in \mathbb{N}}$ are uniformly universally good in $L^{2}$.

Similarly, with $d \geq 1,\left\{\epsilon_{k}\right\}_{k \in \mathbb{N}} \subset \mathbb{N}^{d}$ positive i.i.d with $E\left(\min _{1 \leq j \leq d} 1 / \epsilon_{k, j}\right)<\infty$, $\left\{\gamma_{k}\right\}_{k \in \mathbb{N}} \subset \mathbb{R}^{d}$ bounded i.i.d., and let $\zeta=\chi_{[0,1]^{d}}$. We have

$$
\sup _{t} \prod_{1 \leq j \leq d} \max \left(1,\left|t_{j}\right|\right)|\widehat{\zeta}(t)| \leq 1
$$

Thus, from Zorin-Kranich [43, the averages $F_{n}$ defined by the sequence of transition probability measures $\left\{\delta_{\left(k, k^{2}, \ldots, k^{d}\right)+\gamma_{k}(\omega)} * \zeta_{\epsilon_{k}(\omega)}\right\}_{k \in \mathbb{N}}$ are uniformly universally and variationally good in $L^{2}$.

\section{UNIFORM ESTIMATES}

Estimates for random trigonometric polynomials have been essential in proving convergence of random Fourier series as well as ergodic averages along subsequences and modulated ergodic averages. Paley and Zygmund(1930-32) 30 and Salem and Zygmund (1954) [37] provided the first estimates for trigonometric sums in their study of Fourier series with random signs: $\sum_{k=1}^{\infty} \epsilon_{k} c_{k} e^{i k x}$ where the $\left\{\epsilon_{k}\right\}$ is a Rademacher sequence, and $\left\{c_{k}\right\}$ is a sequence of complex numbers. They were also used to prove convergence of random Fourier and almost periodic series [14, 16, 19, 23, 41 .

In ergodic theory, their study yielded applications to the convergence of averages along subsequences and averages with random weights. Bourgain 9 12] used them to prove pointwise convergence of ergodic averages along polynomial sequences and Bourgain and Wierdl 42 applied them to pointwise convergence of ergodic averages along sequences of primes. Bourgain, Bergelson and Boshenitzan [7] use them to prove pointwise convergence of ergodic averages with random weights as well as Assani [4, 5, Rosenblatt and Wierdl [35, and Cohen and Lin [18. Schneider 39] used them to prove convergence of ergodic averages along perturbed sequences of squares, with integer perturbations.

Estimates on the associated trigonometric polynomials was obtained by means of an estimate on the derivative of those polynomials as in Kahane 26. Cohen and Cuny [16] extended the estimates of Salem and Zygmund to obtain uniform estimates of multidimensional random exponential sums of the form $\sum_{k=1}^{n} X_{k} e^{i\left\langle t, \alpha_{k}\right\rangle}$, where $\left\{X_{n}\right\}$ is a sequence of random variables, $\left\{\alpha_{k}\right\} \subset \mathbb{R}^{d}$ are sequences of real numbers, and $t \in \mathbb{R}^{d}$. Cohen [14] then adapted it to weighted sums of finite transition measures as stated in Theorem 2.3 .

Recent works have also used such estimates to study convergence of power series of isometries with random coefficients, including Assani 6], Boukhari and Weber [8], Cohen and Lin [18, Cohen and Cuny [15, 16] and Cohen [14].

Notation. Henceforth, we denote the inner product for vectors $u, v$ in $R^{d}$ as $\langle u, s\rangle=$ $\sum_{i=1}^{d} u_{i} s_{i}$; the coordinate-wise product as $u . s=\left(u_{1} s_{1}, \ldots, u_{d} s_{d}\right)$, and $|u|=$ $\max _{1 \leq i \leq d}\left|u_{i}\right|$. 
Definition 2.1. Let $(\Omega, \mathcal{F}, P)$ be a probability space, and $\mathcal{B}$ the Borel sigmaalgebra on $\mathbb{R}^{d}, d \geq 1$. A function $\nu: \Omega \times \mathbb{R}^{d} \mapsto \mathbb{C}^{d}$ is a finite complex valued transition measure on $\Omega \times \mathbb{R}^{d}$ if

(i) $\nu(., B)$ is an $\mathcal{F}$-measurable function for any $B \in \mathcal{B}$;

(ii) $\nu(\omega,$.$) is a finite complex valued measure on \mathcal{B}$, for any $\omega \in \Omega$;

(iii) and $E_{\Omega}(|\nu()|)<.\infty$, where $|\nu(\omega)|:=|\nu|\left(\omega, \mathbb{R}^{d}\right)$ denotes the variation norm of the measure $\nu(\omega,$.$) and E_{\Omega} \nu$ denotes the measure, on $\mathcal{B}$, defined by $E_{\Omega} \nu(B)=\int_{\Omega} \nu(\omega, B) d P$, for any $B \in \mathcal{B}$.

Notation. We will say that a sequence of transition measures $\left\{\nu_{k}\right\}$ is bounded if $\sup _{k}\left\|\left|\nu_{k}\right|\right\|_{L^{\infty}(\Omega)}<\infty$.

Definition 2.2. The sequence $\left\{\nu_{k}\right\}_{k \in I}$ of transition measures is independent if for every finite set of Borel measurable simple functions $g_{1}, \ldots, g_{m}$ on $\mathbb{R}^{d}$, and any finite set $k_{1}, k_{2}, \ldots, k_{m}$ of pairwise distinct indices in $I$, the random variables $\left\{\int_{\mathbb{R}^{d}} g_{i}(x) \nu_{k_{i}}(\omega, d x)\right\}_{i=1, \ldots, m}$ are independent.

Given a transition measures $\nu$, its Fourier-Stieljes transform is

$$
\widehat{\nu}_{k}^{\omega}(t)=\int_{\mathbb{R}^{d}} e^{i\langle t, u\rangle} \nu(\omega, d u) .
$$

Uniform estimates that control linear sums of transition measures were considered by Cohen in 14 .

Theorem 2.3 (Theorem 2.8 in [14]). Let $\left\{L_{k}\right\}_{k \in \mathbb{N}}$ be a sequence of positive numbers, $L_{k} \geq 1$, such that $\sum_{n=1}^{\infty} \sum_{m=n+1}^{\infty}\left(1 / L_{n, m}^{2}\right)<\infty$, with $L_{n, m}=\sum_{k=n+1}^{m} L_{k}^{2}$. Let $\left\{\nu_{k}\right\}_{k \in \mathbb{N}}$ be a sequence of independent, finite complex valued transition measure on $\Omega \times \mathcal{B}$ with $\left\|\left|\nu_{k}\right|\right\|_{L^{\infty}(\Omega)}<\infty$, for all $k \geq 1$. Let

$$
P_{n, m}(t)=\sum_{k=n+1}^{m}\left[\int_{\left[-L_{k}, L_{k}\right]^{d}} e^{i\langle t, u\rangle} \nu_{k}(d u)-\int_{\left[-L_{k}, L_{k}\right]^{d}} e^{i\langle t, u\rangle} E_{\Omega} \nu_{k}(d u)\right]
$$

be the sum of the difference of the (truncated) Fourier-Stieljes transform corresponding to the measures $\left\{\nu_{k}(., t)\right\}$ and their expected values. And let $V_{n, m}=$ $\sum_{k=n+1}^{m}\left\|\left|\nu_{k}\right|\right\|_{L^{\infty}(\Omega)}^{2}$. Then, there exists $\epsilon>0$ and $C>0$, independent of $\left\{\nu_{k}\right\}$, such that

$$
\left\|\sup _{m>n} \sup _{T \geq 2} \exp \left(\epsilon \frac{\max _{t \in[-T, T]^{d}}\left|P_{n, m}(\omega, t)\right|^{2}}{V_{n, m} \log \left(L_{n, m}^{2+d / 2} T^{d+2}\right)}\right)\right\|_{L^{1}(\Omega)}<C .
$$

An immediate consequence is the following proposition.

The notation $\varphi(x) \gtrsim|x|$ means there exists a constant $c>0$ such that $\varphi(x) \geq$ $c|x|$. We use $C($.$) or c($.$) to denote functions that depend on the indicated param-$ eter(s), whose values may change in the different instances.

Proposition 2.4. Let $\varphi: \mathbb{R} \rightarrow \mathbb{R}^{+}$be non-decreasing with $\varphi(x) \gtrsim|x|$. Let $\left\{\nu_{k}\right\}_{k \in \mathbb{N}^{r}}$ be independent complex valued transition measures on $\Omega \times \mathcal{B}$, and $\left\{a_{k}\right\}_{k \in \mathbb{N}^{r}}$ a sequence in $(0,1]$. Assume that

$$
\sum_{k \in \mathbb{N}^{r}} a_{k} E_{\Omega}\left|\nu_{k}\right|(|t|>\varphi(|k|))<\infty .
$$


Let $N_{n, m}=\left\{k \in \mathbb{N}^{r}: n<|k| \leq m\right\}$. Then there exists $C: \Omega \rightarrow \mathbb{R}^{+}$finite P-a.e. such that, for all $m$,

$$
\sup _{m>n T>2} \frac{\max _{t \in[-T, T]^{d}}\left|\sum_{k \in N_{n, m}} a_{k}\left(\widehat{\nu}_{k}^{\omega}(t)-\widehat{E_{\Omega} \nu_{k}}(t)\right)\right|^{2}}{1+\sum_{k \in N_{n, m}} a_{k}^{2}\left\|\left|\nu_{k}\right|\right\|_{L^{\infty}(\Omega)}^{2} \log (\max (\varphi(m), T))} \leq C(w) .
$$

In particular, if $\left\{\nu_{k}\right\}_{k \in \mathbb{N}^{r}}$ are bounded then

$$
\sup _{m>n} \sup _{T>2} \frac{\max _{t \in[-T, T]^{d}}\left|\sum_{k \in N_{n, m}} a_{k}\left(\widehat{\nu}_{k}^{\omega}(t)-{\widehat{E} \Omega \nu_{k}}(t)\right)\right|^{2}}{\left[\sum_{k \in N_{n, m}} a_{k}^{2}\right] \log (\max (\varphi(m), T))} \leq C(w) .
$$

Proof. Theorem 2.3 can be applied to the transition measures $w_{k}=a_{k} \nu_{k}$, now indexed over $k \in \mathbb{N}^{r}$, by simple re-numbering of the index set, with $L_{k}=\varphi(|k|)$. We have

$$
L_{n, m}=\sum_{n<|k| \leq m} L_{k}^{2} \sim \sum_{n<j \leq m} j^{r-1} \varphi^{2}(j) \lesssim m^{r} \varphi(m)^{2} \lesssim \varphi(m)^{r+2}
$$

and

$$
V_{n, m}=\sum_{n<|k| \leq m}\left\|\left|w_{k}\right|\right\|_{L^{\infty}(\Omega)}^{2}=\sum_{n<|k| \leq m} a_{k}^{2}\left\|\left|\nu_{k}\right|\right\|_{L^{\infty}(\Omega)}^{2} .
$$

Let $J_{n}=[-\varphi(n), \varphi(n)]^{d}$ and $\widehat{D \nu}_{k}(\omega, t)=a_{k}\left(\widehat{\nu}_{k}(t)-{\widehat{E} \nu_{k}}_{k}(t)\right)$.

Decompose $\sum_{k \in N_{n, m}} \widehat{D \nu}_{k}(\omega, t)=P_{n, m}+Q_{n, m}$, where

$$
P_{n, m}(\omega, t)=\sum_{k \in N_{n, m}} a_{k}\left[\int_{J_{|k|}} e^{i\langle t, u\rangle} \nu_{k}(\omega, d u)-\int_{J_{|k|}} e^{i\langle t, u\rangle} E_{\Omega} \nu_{k}(d u)\right],
$$

and

$$
Q_{n, m}(\omega, t)=\sum_{k \in N_{n, m}} a_{k}\left[\int_{J_{|k|}^{c}} e^{i\langle t, u\rangle} \nu_{k}(\omega, d u)-\int_{J_{|k|}^{c}} e^{i\langle t, u\rangle} E_{\Omega} \nu_{k}(d u)\right] .
$$

From Theorem 2.3 there is $C_{1}: \Omega \rightarrow \mathbb{R}^{+}$finite $P$-a.e. such that

$$
\begin{aligned}
\max _{t \in[-T, T]^{d}}\left|P_{n, m}(\omega, t)\right|^{2} & \lesssim C_{1}(\omega) V_{n, m} \log \left(L_{n, m}^{2+d / 2} T^{d+2}\right) \\
& \sim c(d) C_{1}(\omega)\left[\sum_{k \in N_{n, m}} a_{k}^{2}\left\|\left|\nu_{k}\right|\right\|_{L^{\infty}(\Omega)}^{2}\right] \log (\max (\varphi(m), T)) .
\end{aligned}
$$

We also have

$$
\left|Q_{n, m}(\omega, t)\right| \lesssim \sum_{k \in N_{n, m}} a_{k}\left[\left|\nu_{k}\right|\left(\omega, J_{|k|}^{c}\right)+E_{\Omega}\left|\nu_{k}\right|\left(J_{|k|}^{c}\right)\right] .
$$

By assumption on $\left\{\nu_{k}\right\}$, there exists $C_{2}(\omega) \in L^{1}(\Omega)$ such that

$$
\sup _{m>n \geq 1} \sup _{t}\left|Q_{n, m}(\omega, t)\right| \lesssim C_{2}(\omega) .
$$

Combining both estimates, 2.4.1) is obtained. 
Example 2.5. Let $\left\{n_{k}\right\}_{k \in \mathbb{N}^{r}} \subset\left(\mathbb{R}^{+}\right)^{d} ;\left\{\gamma_{k}\right\}_{k \in \mathbb{N}^{r}}$ be independent bounded random vectors such that $\sup _{k}\left\|\gamma_{k}\right\|_{L^{\infty}}=B<\infty$; and let $\left\{X_{k}\right\}_{k \in \mathbb{N}^{r}}$ be i.i.d complex valued random variables, independent of $\left\{\gamma_{k}\right\}_{k \in \mathbb{N}^{r}}$, and let $\nu_{k}(\omega, A)=X_{k}(\omega) \delta_{n_{k}+\gamma_{k}(\omega)}(A)$, $A \in \mathcal{D}$. Now

$$
\begin{aligned}
P_{m}(\omega, t) & =\sum_{k \in[1, m]^{r}}\left(\widehat{\nu_{k}^{\omega}}(t)-\widehat{E_{\Omega} \nu_{k}}(t)\right) \\
& =\sum_{k \in[1, m]^{r}} e^{i\left\langle t, n_{k}\right\rangle}\left(X_{k}(\omega) e^{i\left\langle t, \gamma_{k}(\omega)\right\rangle}-E_{\Omega}\left(X_{1}\right) E_{\Omega}\left(e^{i\left\langle t, \gamma_{k}\right\rangle}\right)\right) .
\end{aligned}
$$

If $\sup _{k}\left|n_{k}\right| / 2^{|k|^{r \beta}}=c<\infty$, for some $0<\beta<1$, using $\varphi(x)=(1+c) 2^{|x|^{r \beta}}$, the assumption of Proposition 2.4 is satisfied by a tail distribution condition on the $\gamma_{k}$ 's

$$
\sum_{k \in \mathbb{N}^{r}} E_{\Omega}\left|\nu_{k}^{\omega}\right|\left(|t|>(1+c) 2^{|k|^{r \beta}}\right) \leq E_{\Omega}\left(\left|X_{1}\right|\right) \sum_{k \in \mathbb{N}^{r}} P\left(\left|\gamma_{k}(\omega)\right|>2^{|k|^{r \beta}}\right)<\infty .
$$

Then, by Proposition 2.4, there exists $C(\omega) \in L^{1}(\Omega)$, such that

$$
\sup _{m \geq 1} \sup _{T \geq 2} \max _{t \in[-T, T]^{d}} \frac{\left|P_{m}(\omega, t)\right|^{2}}{1+m^{r} \log \left(\max \left(T, 2^{m^{r \beta}}\right)\right)}=C(\omega)<\infty .
$$

In particular, for $\widehat{D}_{n}(\omega, t)=\frac{1}{n^{r}} P_{n}(\omega, t)$ and $T \leq 2^{n^{r \beta}}$, we obtain

$$
\max _{t \in[-T, T]^{d}}\left|\widehat{D}_{n}(\omega, t)\right|^{2} \lesssim C(w) \frac{\log \left(\max \left(T, 2^{n^{r \beta}}\right)\right)}{n^{r}} \leq C(w) \frac{1}{n^{r(1-\beta)}} .
$$

\section{Applichtions to ergodic theory}

In this section, $\left\{\nu_{k}\right\}_{k \in \mathbb{N}^{r}}$ denotes a sequence of independent transition measures on $\Omega \times \mathcal{B}, V_{m}=\sum_{k \in[1, m]^{r}}\left\|\left|\nu_{k}\right|\right\|_{L^{\infty}(\Omega)}^{2}$ and $b_{m}=\sum_{k \in[1, m]^{r}}\left\|\left|\nu_{k}\right|\left(., \mathbb{R}^{d}\right)\right\|_{L^{\infty}(\Omega)}$. The averages

$$
K_{n}^{\omega} f(x)=K_{n} f(x)=\frac{1}{b_{n}} \sum_{k \in[1, n]^{r}} \nu_{k}^{\omega}(f)(x)
$$

have associated kernels

$$
\widehat{K}_{n}^{\omega}(t)=\widehat{K}_{n}(t)=\frac{1}{b_{n}} \sum_{k \in[1, n]^{r}} \widehat{\nu}_{k}(\omega, t)=\frac{1}{b_{n}} \sum_{k \in[1, n]^{r}} \int e^{i\langle t, u\rangle} \nu_{k}(\omega, d u) .
$$

Next, consider the averages defined by their expected values

$$
E_{n} f(x)=E_{\Omega}\left(K_{n} f(x)\right)=\frac{1}{b_{n}} \sum_{k \in[1, n]^{r}} E_{\Omega} \nu_{k} f(x)
$$

and its associated kernel

$$
\widehat{E}_{n}(t)=E_{\Omega}\left(\widehat{K}_{n}(t)\right)=\frac{1}{b_{n}} \sum_{k \in[1, n]^{r}} E_{\Omega}\left(\widehat{\nu}_{k}^{\omega}\right)(t)=\frac{1}{b_{n}} \sum_{k \in[1, n]^{r}} \int e^{i\langle t, u\rangle} E_{\Omega} \nu_{k}(d u) .
$$

Let $D_{n} f(x)=K_{n} f(x)-E_{n} f(x)$ and $\widehat{D}_{n}(t)=\widehat{K}_{n}(t)-\widehat{E}_{n}(t)$ denote the corresponding centered averages and their kernels. 
Note 3.1. A family $\left\{T_{t}\right\}_{t \in\left(\mathbb{R}^{+}\right)^{d}}$ is a continuous parameter semi-flow of isometries acting on a Hilbert space $H$ or an $\left(\mathbb{R}^{+}\right)^{d}$-action, if for all $\mathbf{x} \in H, T_{0} \mathbf{x}=\mathbf{x}$, $T_{s} T_{t} \mathbf{x}=T_{s+t} \mathbf{x}$ for $s, t \in\left(\mathbb{R}^{+}\right)^{d}$, the map $t \rightarrow\left\langle T_{t} \mathbf{x}, \mathbf{x}\right\rangle$ is continuous, and $T_{t}$ is an isometry for each $t \geq 0$.

A classical generalization of Stone's Theorem 34 provides a spectral representation for unitary actions of $\mathbb{R}^{d}$. That is, if $\left\{T_{t}\right\}_{t \in \mathbb{R}^{d}}$ is a $\mathbb{R}^{d}$-action by unitary operators on a Hilbert space $H$, then for any $\mathbf{x} \in H$, there is a positive finite measure $\mu_{\mathbf{x}}$ on $\mathbb{R}^{d}$, called its spectral measure, such that for any $s \in \mathbb{R}^{d}$, $\left\langle T_{s} \mathbf{x}, \mathbf{x}\right\rangle=\int_{\mathbb{R}^{d}} e^{i\langle s, t\rangle} d \mu_{\mathbf{x}}(t)$.

Such result extends to $\mathbb{R}^{d}$ isometry actions via the following unitary dilation theorem (Principal Theorem in Appendix to Riesz-Nagy Functional Analysis [34]).

Theorem 3.2 (Riesz-Nagy, [34]). Let $\left\{T_{t}\right\}_{t \in \mathbb{R}^{d}}$ be a representation of $\mathbb{R}^{d}$ by isometries on a Hilbert space $H$. There exist a Hilbert space $H_{2} \supset H$ and an $\mathbb{R}^{d}$ action $\left\{U_{t}\right\}_{t \in \mathbb{R}^{d}}$ by unitary operators on $H_{2}$, such that, if $\pi: H_{2} \rightarrow H$ is the orthogonal projection, then $\pi U_{t} \mathbf{x}=T_{t} \mathbf{x}$, for all $t \in \mathbb{R}^{d}$ and $\mathbf{x} \in H$.

Remark. Norm estimates can be extended to actions by contractions in the case $d=1$, via Sz. -Nagy unitary dilation theorem (Szökefalvi-Nagy and Foias [40]). This still may hold for $d=2$ but not in higher dimensions. Parrott 31] gave an example where the dilation theorem is no longer true in the case of $\mathbb{N}^{3}$ actions by contractions.

Remark. For actions by semigroup of isometries, the spectral representation allows us to estimate norms for operators defined by a finite measure on the semigroup. With the notation of Riesz-Nagy theorem and the spectral measure, if $\nu$ a finite measure on $\left(\mathbb{R}^{+}\right)^{d}$, by standard arguments we conclude

$$
\left\|\int_{\mathbb{R}^{d}} T_{t} \mathbf{x} \nu(d t)\right\|_{H}^{2} \leq\left\|\int_{\mathbb{R}^{d}} U_{t} \mathbf{x} \nu(d t)\right\|_{H^{\prime}}^{2}=\int_{\mathbb{R}^{d}}|\widehat{\nu}(t)|^{2} d \mu_{\mathbf{x}}(t) .
$$

Notation. For any positive real number $v$, abusing notation we write $K_{v} f=K_{\lfloor v\rfloor} f$, $D_{v} f=D_{\lfloor v\rfloor} f, b_{v}=b_{\lfloor v\rfloor}, V_{v}=V_{\lfloor v\rfloor}$, etc.. Also, $\varphi$ is a continuous strictly increasing positive function on $\mathbb{R}$, and $\psi$ its inverse $\psi(y)=\inf \{x \geq 0: \varphi(x)>y\}$.

Theorem 3.3. Let $\left\{\nu_{k}\right\}_{k \in \mathbb{N}^{r}}$ be a sequence of independent, finite complex valued transition measures on $\Omega \times \mathcal{B}$. Let $\varphi$ be a non-decreasing positive function such that $\varphi(|x|) \gtrsim|x|$, and $\psi$ denotes its inverse. Assume that
(a) $\sup _{n} \frac{1}{b_{n}} \sum_{k \in[1, n]^{r}}\left|\nu_{k}(\omega)\right| \in L^{1}(\Omega)$,
(b) $\sum_{k \in \mathbb{N}^{r}} E_{\Omega}\left|\nu_{k}\right|(|t|>\varphi(|k|))<\infty$, and
(c) $c(\rho)=\sum_{n \geq 1} \frac{1+V_{\rho^{n}} \log \left(\varphi\left(\rho^{n}\right)\right)}{b_{\rho^{n}}^{2}}<\infty$, for all $\rho>1$.

Then, there exists $0<C \in L^{\infty}(\Omega)$ such that, for any $\rho>1$ and any probability space $(X, \mathcal{D}, m)$ with any continuous semi-flow of isometries $\left\{T_{t}\right\}_{t \in\left(\mathbb{R}^{+}\right)^{d}}$ acting on it, if $f \in L^{2}(X)$,

$$
\left\|\sqrt{\sum_{n=1}^{\infty}\left|D_{\rho^{n}} f\right|^{2}}\right\|_{2}<C(\omega) \tilde{c}(\rho)\|f\|_{(\log \psi)^{+} L^{2}},
$$

where $\tilde{c}(\rho)^{2}=c(\rho) \vee\left(\log _{2} \rho\right)^{-1}$. 
Proof.

$$
\begin{array}{rl}
\int_{\mathbb{R}^{d}} \sum_{n=1}^{\infty}\left|D_{\rho^{n}} f\right|^{2} & d m \leq \sum_{n=1}^{\infty} \int_{\mathbb{R}^{d}}\left|\widehat{D}_{\rho^{n}}(t)\right|^{2} d \mu_{f} \\
= & \sum_{n=1}^{\infty} \int_{|t| \leq \varphi\left(\rho^{n}\right)}\left|\widehat{D}_{\rho^{n}}(t)\right|^{2} d \mu_{f}+\sum_{n=1}^{\infty} \int_{|t|>\varphi\left(\rho^{n}\right)}\left|\widehat{D}_{\rho^{n}}(t)\right|^{2} d \mu_{f}=\mathrm{I}+\mathrm{II} .
\end{array}
$$

By assumption (b) and Proposition 2.4.

$$
C_{1}(\omega)=\sup _{\rho>1, n} \frac{\max _{|t| \leq \varphi\left(\rho^{n}\right)}\left|\widehat{D}_{\rho^{n}}(t)\right|^{2} b_{\rho^{n}}^{2}}{1+V_{\rho^{n}} \log \left(\varphi\left(\rho^{n}\right)\right)}
$$

is finite $\mathrm{P}-$ a.e. Therefore, by assumption (c),

$$
I \lesssim C_{1}(\omega) \sum_{n=1}^{\infty} \frac{1+V_{\rho^{n}} \log \left(\varphi\left(\rho^{n}\right)\right)}{b_{\rho^{n}}^{2}}\|f\|_{2}^{2}=C_{1}(\omega) c(\rho)\|f\|_{2}^{2} .
$$

For the second term II, by assumption (a)

$$
\sup _{n} \sup _{t}\left|\widehat{D}_{n}(t)\right| \leq \sup _{n} \frac{1}{b_{n}} \sum_{k \in[1, n]^{r}}\left|\nu_{k}(\omega)\right|=C_{2}(\omega) \in L^{1}(\Omega) .
$$

Thus

$$
\begin{aligned}
\mathrm{II} & =\sum_{n=1}^{\infty} \sum_{k \geq n} \int_{\varphi\left(\rho^{k}\right)<|t| \leq \varphi\left(\rho^{(k+1)}\right)}\left|\widehat{D}_{\rho^{n}}(t)\right|^{2} d \mu_{f} \leq C_{2}^{2}(\omega) \sum_{k=1}^{\infty} k \int_{\varphi\left(\rho^{k}\right)<|t| \leq \varphi\left(\rho^{(k+1)}\right)} d \mu_{f} \\
& \leq \frac{C_{2}^{2}(\omega)}{\log _{2} \rho} \sum_{k=1}^{\infty} \int_{\varphi\left(\rho^{k}\right)<|t| \leq \varphi\left(\rho^{(k+1)}\right)} \log _{2} \psi(|t|) d \mu_{f} \lesssim \frac{C_{2}^{2}(\omega)}{\log _{2} \rho} \int(\log \psi)^{+}|t| d \mu_{f} .
\end{aligned}
$$

Combining the estimates for both terms I and II, the proposition is proven.

We are interested in the cases where $\left\{\nu_{k}\right\}$ are transition probability measures, thus condition (a) in this theorem is immediately satisfied. Condition (c) is fulfilled by the choice of growth function $\varphi$. Thus, the restriction on the estimate for the square function on $\left\{D_{\rho^{n}} f\right\}$ comes from the distribution of the transition probability measures $\left\{\nu_{k}\right\}$.

Proposition 3.4. Let $\left\{\nu_{k}\right\}_{k \in \mathbb{N}^{r}}$ be a sequence of independent, bounded transition measures on $\Omega \times \mathcal{B}$ such that, for some $0<\beta<1$ and some constant $c>0$,

$$
\sum_{k \in \mathbb{N}^{r}} E_{\Omega}\left|\nu_{k}\right|\left(|t|>c 2^{|k|^{r \beta}}\right)<\infty .
$$

Then there exists $0<C \in L^{\infty}(\Omega)$ and a constant $c(\rho, \beta)$, such that, for any probability space $(X, \mathcal{D}, m)$ with any continuous semi-flow of positive isometries $\left\{T_{t}\right\}_{t \in\left(R^{+}\right)^{d}}$ acting on it, if $f \in L^{2}(X)$,

$$
\left\|\sqrt{\sum_{n=1}^{\infty}\left|D_{\rho^{n}} f\right|^{2}}\right\|_{2}^{2}<C(\omega) c(\rho, \beta)\|f\|_{(\log \log )+L^{2}} \text { for any } \rho>1 .
$$


Proof. Choosing $\varphi(x)=c 2^{x^{r \beta}}$, for $x \geq 0$, it suffices to check the assumptions of Theorem 3.3. Since $\left\{\nu_{k}\right\}_{k \in \mathbb{N}^{r}}$ are bounded transition measures, $\sup _{\omega \in \Omega} \sup _{k}\left|\nu_{k}(\omega)\right|$ $=K<\infty$. Thus, $b_{n} \sim n^{r}$ and $V_{n} \sim n^{r}$ and

$$
\sup _{\omega \in \Omega} \sup _{n} \frac{1}{b_{n}} \sum_{k \in[1, n]^{r}}\left|\nu_{k}(\omega)\right| \sim K
$$

satisfying assumption (a). Assumption (b) becomes the assumption of this proposition, and assumption (c) is satisfied for $\beta \in(0,1)$

$$
\begin{aligned}
c(\rho, \beta) & =\sum_{n \geq 1} \frac{1+V_{\rho^{n}} \log \left(\varphi\left(\rho^{n}\right)\right)}{b_{\rho^{n}}^{2}} \sim \sum_{n \geq 1} \frac{\rho^{r n} \log \left(2^{\rho^{r \beta n}}\right)}{\rho^{2 r n}} \\
& =\sum_{n \geq 1} \frac{1}{\rho^{(1-\beta) r n}}<\infty \text { for all } \rho>1 .
\end{aligned}
$$

After the square function result along exponential subsequences $I(\rho)=\left\{\rho^{n}\right\}$ is obtained for all $\rho>1$, Theorem 3.3 or Proposition 3.4 one is tempted to prove a variational inequality for averages with kernels $\widehat{K}_{n}$. But without additional control on the decay of the kernels, this approach may fail to be fruitful. However, this result is enough for universal pointwise convergence of the averages. The steps for proving convergence were inspired by [35] and related works.

Proposition 3.5 (Properties of variation norms).

(a) For $s \geq 1,\|\cdot\|_{v(s)}$ is a semi-norm;

(b) $\left\|x_{n}\right\|_{v(s)} \leq 2\left(\sum_{n=1}^{\infty}\left|x_{n}\right|^{s}\right)^{1 / s}$; and

(c) $\left\|x_{n}\right\|_{v(s)} \leq 2 \sum_{k}\left\|\left\{x_{n}: n_{k} \leq n<n_{k+1}\right\}\right\|_{v(s)}$ for any sequence $n_{k}$ such that $x_{n_{k}}=0$ for all $k$.

A proof of these properties can be found in [17] along with some discussion of the applications of the variation norm to ergodic theory, see also [24,25].

A complex valued transition measure $\nu$ is dominated by a positive transition measure, defined by its variation norm, which by abusing notation we denote by $|\nu|$,

$$
|\nu|(\omega, B)=|\nu(\omega, B)|=\text { variation norm of } \nu(\omega, .) \text { restricted to the set } B, B \in \mathcal{B} \text {. }
$$

Continuing abusing notation, we denote

$$
\left|K_{n}^{\omega}\right| f(x)=\frac{1}{b_{n}} \sum_{k \in[1, n]^{r}}\left|v_{k}\right|^{\omega} f(x) \text { and }\left|E_{n}\right| f(x)=\frac{1}{b_{n}} \sum_{k \in[1, n]^{r}} E_{\Omega}\left|v_{k}\right| f(x) .
$$

Theorem 3.6. With the assumptions and notation of Theorem 3.3.

(a) $\left\{K_{n}-E_{\Omega} K_{n}\right\}_{n \in I(\rho)}$ are uniformly universally good in $(\log \psi)^{+} L^{2}$ for any $\rho>1$.

(b) If for some $\rho>1$, the averages $\left\{E_{n}\right\}_{n \in I(\rho)}$ are universally good in $L^{2}$, then the averages $\left\{K_{n}\right\}_{n \in I(\rho)}$ are uniformly universally good in $(\log \psi)^{+} L^{2}$.

(c) If for some $\rho>1,\left\{E_{n}\right\}_{n \in I(\rho)}$ are variationally good in $L^{2}$, then $\left\{K_{n}\right\}_{n \in I(\rho)}$ are uniformly variationally good in $(\log \psi)^{+} L^{2}$. 
(d) Assume $\left\{E_{n}\right\}_{n \in I(\rho)}$ are universally good in $L^{2}$ for all $\rho>1$, and

$$
\lim _{\rho \mapsto 1} \sup _{n} b_{\rho^{n+1}} / b_{\rho^{n}}=1 .
$$

If $\left\{\nu_{k}\right\}_{k \in \mathbb{N}^{r}}$ are real-valued positive transition measures or $\left\{\left|E_{n}\right|\right\}_{n \in I(\rho)}$ are also universally good in $L^{2}$, then $\left\{K_{n}\right\}_{n \in \mathbb{N}}$ is uniformly universally good in $(\log \psi)^{+} L^{2}$.

Proof. By Theorem 3.3. there exists $0<C(\omega) \in L^{\infty}(\Omega)$ and $c(\rho) \in(0, \infty)$ such that, for any probability space $(X, \mathcal{D}, m)$, any continuous semi-flow of positive isometries $\left\{T_{t}\right\}_{t \in\left(\mathbb{R}^{+}\right)^{d}}$ in $L^{2}(X)$,

$$
\left\|\sqrt{\sum_{n}\left|D_{\rho^{n}} f\right|^{2}}\right\|_{2} \leq C(\omega) c(\rho)\left[\int(\log \psi)^{+}|t| d \mu_{f}\right]^{1 / 2} .
$$

Thus parts (a) and (b) are an immediate consequence of this result. Moreover, letting $\Omega^{\prime} \subset \Omega$ of probability 1 , such that $C(\omega)<\infty$ for $\omega \in \Omega^{\prime}$, then for any probability space $(X, \mathcal{D}, m)$, any continuous semi-flow of positive isometries $\left\{T_{t}\right\}_{t \in\left(\mathbb{R}^{+}\right)^{d}}$ in $L^{2}(X), \lim _{n \rightarrow \infty} K_{\rho^{n}} f(x)-E_{\rho^{n}} f(x)=0$ a.e. for any $\rho>1$.

Part (c): Since $\left\{E_{n}\right\}_{n \in I(\rho)}$ are variationally good in $L^{2}$, there exists $s>2$ and $c^{\prime}(s, \rho)>0$ such that \|\|$E_{\rho^{n}} f\left\|_{v(s)}\right\|_{2} \leq c^{\prime}(s, \rho)\|f\|_{2}$. For $\omega \in \Omega^{\prime}$, by Proposition 3.5.

$$
\begin{aligned}
\|\| K_{\rho^{n}} f\left\|_{v(s)}\right\|_{2} & \leq\|\| E_{\rho^{n}} f\left\|_{v(s)}\right\|_{2}+\|\| D_{\rho^{n}} f\left\|_{v(s)}\right\|_{2} \\
& \leq c^{\prime}(s, \rho)\|f\|_{2}+2\left\|\sqrt{\sum_{n}\left|D_{\rho^{n}} f\right|^{2}}\right\|_{2} \\
& \leq\left(c^{\prime}(s, \rho)+2 C(\omega) c(\rho)\right)\left[\int(\log \psi)^{+}|t| d \mu_{f}\right]^{1 / 2} .
\end{aligned}
$$

Part (d):

Case $1\left(\left\{\nu_{k}\right\}_{k \in \mathbb{N}^{r}}\right.$ are real-valued positive transition measures). In this case, since $T_{t}$ 's are positive operators, $K_{n} f$ are themselves positive operators. Consider the sequence of $\rho_{i}=2^{1 / i}$ decreasing to 1 . Since $\left\{E_{n}\right\}_{n \in I(\rho)}$ are universally good in $L^{2}$ for all $\rho>1$, and by (b), for any $\omega \in \Omega^{\prime}$ and any $i, K_{\rho_{i}^{n}} f$ converges almost everywhere for any $f \in(\log \psi)^{+} L^{2}$. It suffices to consider $0 \leq f \in(\log \psi)^{+} L^{2}$, a.e.. Notice that with our choice of $\rho_{i}, \lim _{n \rightarrow \infty} K_{2^{n}}^{\omega} f(x)=\lim _{n \rightarrow \infty} K_{\rho_{i}^{n}}^{\omega} f(x)=$ $\lim _{n \rightarrow \infty} E_{2^{n}} f(x)$ a.e., for any $i \geq 1$, and so we denote this limit as $L f(x)$. Note that this limit does not depend on $\omega$. Letting $M_{i}=\sup _{n} \frac{b_{i}{ }^{n+1}}{b_{\rho_{i}^{n}}}$; for $\rho_{i}^{n} \leq u<\rho_{i}^{n+1}$, we have

$$
\frac{1}{M_{i}} K_{\rho_{i}^{n}}^{\omega} f(x) \leq \frac{b_{\rho_{i}^{n}}}{b_{\rho_{i}^{n+1}}} K_{\rho_{i}^{n}}^{\omega} f(x) \leq K_{u}^{\omega} f(x) \leq \frac{b_{\rho_{i}^{n+1}}}{b_{\rho_{i}^{n}}} K_{\rho_{i}^{n+1}}^{\omega} f(x) \leq M_{i} K_{\rho_{i}^{n+1}}^{\omega} f(x) .
$$

Then

and since $M_{i} \underset{i \rightarrow \infty}{\longrightarrow} 1$,

$$
\frac{1}{M_{i}} L f(x) \leq \liminf _{u \rightarrow \infty} K_{u}^{\omega} f(x) \leq \limsup _{u \rightarrow \infty} K_{u}^{\omega} f(x) \leq M_{i} L f(x),
$$

$$
\lim _{u \rightarrow \infty} K_{u} f(x)=L f(x) \text { a.e.. }
$$

A similar relationship can be established for the averages $E_{n} f$, thus $L f(x)=$ $\lim _{n \rightarrow \infty} K_{n}^{\omega} f(x)=\lim _{n \rightarrow \infty} E_{n} f(x)$ a.e.. 
Case 2 (General transition measures). The conditions of this theorem on the transition measures $\left\{\nu_{k}\right\}_{k \in \mathbb{N}^{r}}$ are actually conditions on $\left\{\left|\nu_{k}\right|\right\}_{k \in \mathbb{N}^{r}}$. Therefore, if both $\left\{E_{\rho^{n}}\right\}$ and $\left\{\left|E_{\rho^{n}}\right|\right\}$ are universally good in $L^{2}$ for any $\rho>1$, the results (a), (b) and (d) part (i) apply to the averages $\left\{\left|K_{n}^{\omega}\right| f\right\}$ and thus both $\left\{\left|K_{n}\right|\right\}$ and $\left\{K_{\rho^{n}}\right\}$ are uniformly universally good in $(\log \psi)^{+} L^{2}$ (for any $\rho>1$ ).

As before, consider the sequence of $\rho_{i}=2^{1 / i}$, decreasing to 1 . Let $\Omega^{\prime \prime} \subset \Omega$ of probability 1 , such that, for any $\omega \in \Omega^{\prime \prime},\left\{\left|K_{n}^{\omega}\right|\right\}$ and $\left\{K_{\rho_{i}^{n}}^{\omega}\right\}$ are universally good in $(\log \psi)^{+} L^{2}$ (for all $i$ ). Again, it suffices to consider $0 \leq f \in(\log \psi)^{+} L^{2}$, and we have $\lim _{n \rightarrow \infty} E_{2^{n}} f(x)=\lim _{n \rightarrow \infty} K_{2^{n}}^{\omega} f(x)=\lim _{n \rightarrow \infty} K_{\rho_{i}^{n}}^{\omega} f(x)=L f(x)$ a.e., for any $i \geq 1$. For $\left\lfloor\rho_{i}^{n}\right\rfloor \leq u<\left\lfloor\rho_{i}^{n+1}\right\rfloor$,

$$
\begin{aligned}
\left|K_{u}^{\omega} f(x)-K_{\rho_{i}^{n}}^{\omega} f(x)\right| & \leq \frac{b_{u}-b_{\rho_{i}^{n}}}{b_{u}}\left|K_{\rho_{i}^{n}}^{\omega} f(x)\right|+\frac{1}{b_{u}} \sum_{l:\left\lfloor\rho_{i}^{n}\right\rfloor<|l| \leq u}\left|\nu_{l}\right|^{\omega} f(x) \\
& \leq \frac{b_{\rho_{i}^{n+1}}-b_{\rho_{i}^{n}}}{b_{\rho_{i}^{n}}}\left|K_{\rho_{i}^{n}}^{\omega} f(x)\right|+\left|\frac{b_{\rho_{i}^{n+1}}}{b_{\rho_{i}^{n}}}\right| K_{\rho_{i}^{n+1}}^{\omega}|f(x)-| K_{\rho_{i}^{n}}^{\omega}|f(x)| \\
\leq & \left(\frac{b_{\rho_{i}^{n+1}}}{b_{\rho_{i}^{n}}}-1\right)\left(\left|K_{\rho_{i}^{n}}^{\omega}\right| f(x)+\left|K_{\rho_{i}^{n+1}}^{\omega}\right| f(x)\right) \\
& +|| K_{\rho_{i}^{n+1}}^{\omega}|f(x)-| K_{\rho_{i}^{n}}^{\omega}|f(x)| .
\end{aligned}
$$

Since $\lim _{i \rightarrow \infty} \sup _{n} \frac{b_{i}{ }_{i}^{n+1}}{b_{\rho_{i}^{n}}}=1$ and $\left\{\left|K_{n}^{\omega}\right|\right\}$ is universally good in $(\log \psi)^{+} L^{2}$, $\lim _{i \rightarrow \infty} \lim \sup _{u \rightarrow \infty}\left|K_{u}^{\omega} f(x)-K_{\rho_{i}^{n}}^{\omega} f(x)\right|=0$ a.e.. thus $\lim _{u \rightarrow \infty} K_{u}^{\omega} f(x)=L f(x)$ a.e., establishing that $\left\{K_{n}\right\}$ are uniformly universally good in $(\log \psi)^{+} L^{2}$.

Remark. Note that if the transition measures $\left\{\nu_{k}\right\}$ are complex valued with $E_{\Omega} \nu_{k}=$ 0, then, uniformly, $K_{n} f \rightarrow 0$ a.s. as $n \underset{n \in I(\rho)}{\longrightarrow} \infty$, for $f \in(\log \psi)^{+} L^{2}$.

Remark. The result of this theorem can be improved to hold in $L^{2}$ with an additional requirement on the $D_{n}$ kernels. See Section 4 .

Proof of Theorem 1.8. It follows as a corollary of Proposition 3.4 and 3.6 noticing that, when $\left\{\gamma_{k}\right\}$ are i.i.d., then $E_{n} f=A_{n}\left(E_{\Omega} T_{\gamma_{e}} f\right)$ (for any $e \in \mathbb{N}^{r}$ ).

Example 3.7 (Essentially the proof of Corollary 1.9) . Consider again Example 2.5 with $\left\{n_{k}\right\}_{k \in \mathbb{N}}$ an integer valued polynomial sequence $\left(n_{k}=p(k)\right.$ for some integercoefficient polynomial $p(x)$ ), or any universally good sequence in $L^{2}$. Let $\left\{\gamma_{k}\right\}_{k \in \mathbb{N}}$, $\left\{X_{k}\right\}_{k \in \mathbb{N}}$ be independent sequences of i.i.d. real-valued variables with $E_{\Omega}\left(\left|\gamma_{1}\right|\right)<$ $\infty$ and $E_{\Omega}\left(\left|X_{1}\right|\right)<\infty$. Let $\nu_{k}(\omega,)=.X_{k}(\omega) \delta_{n_{k}+\gamma_{k}(\omega)}$. Then

$$
\begin{aligned}
\sum_{k \in \mathbb{N}} E_{\Omega}\left|\nu_{k}\right|\left(|t|>c 2^{|k|^{\beta}}\right) & =\sum_{k \in \mathbb{N}} E_{\Omega}\left(\left|X_{k}\right|\right) P\left(\left|\gamma_{k}\right|>c 2^{k^{\beta}}\right) \\
& <E_{\Omega}\left(\left|X_{1}\right|\right) E_{\Omega}\left(\max \left(1, \log \left|\gamma_{1}\right| / c\right)^{1 / \beta}\right) \\
& <E_{\Omega}\left(\left|X_{1}\right|\right) \frac{E_{\Omega}\left(\max \left(1,\left|\gamma_{1}\right| / c\right)\right.}{\beta^{1 / \beta}}<\infty .
\end{aligned}
$$

Also

$$
E_{\Omega} \nu_{k} f=E_{\Omega}\left(X_{k}\right) T_{n_{k}} E_{\Omega} T_{\gamma_{k}(\omega)} f=T_{n_{k}} g,
$$


where $g=E_{\Omega}\left(X_{k}\right) E_{\Omega} T_{\gamma_{1}} f \in L^{2}(X)$ if $f \in L^{2}(X)$. Thus $E_{n} f=A_{n} g,\left|E_{n}\right| f=A_{n} h$, $h=\left|E_{\Omega}\left(X_{k}\right)\right| E_{\Omega} T_{\gamma_{1}} f$, and therefore, $E_{n}$ and $\left|E_{n}\right|$ are universally good in $L^{2}$. Thus, by Theorem [3.6, the averages $\frac{1}{n} \sum_{k=1}^{n} X_{k}(\omega) T_{n_{k}+\gamma_{k}(\omega)} f(x)$ are uniformly universally good in $(\log \log )^{+} L^{2}$. Also, from the proof,

$$
\lim _{n \rightarrow \infty} \frac{1}{n} \sum_{k=1}^{n} X_{k}(\omega) T_{n_{k}+\gamma_{k}(\omega)} f(x)=E_{\Omega}\left(X_{1}\right) \lim _{n \rightarrow \infty} A_{n}\left(E_{\Omega} T_{\gamma_{1}} f\right)(x) .
$$

\section{Smoother AVERAGeS}

We now consider the particular example of averages $F_{n}^{\omega} f(x)$ defined in (1.11.1). Their corresponding kernels

$$
\widehat{F}_{n}^{\omega}(t)=\frac{1}{n^{r}} \sum_{k \in[1, n]^{r}} \widehat{\zeta}\left(t . \epsilon_{k}(\omega)\right) e^{i\left\langle t,\left(n_{k}+\gamma_{k}(\omega)\right)\right\rangle}
$$

decompose as $\widehat{F}_{n}^{\omega}(t)=\widehat{E}_{n}(t)+\widehat{D}_{n}^{\omega}(t)$ where $\widehat{E}_{n}(t)=E_{\Omega} \widehat{F}_{n}(t)$ and

$$
\widehat{D}_{n}^{\omega}(t)=\frac{1}{n^{r}} \sum_{k \in[1, n]^{r}}\left[e^{i\left\langle t, \gamma_{k}(\omega)\right\rangle} \widehat{\zeta}\left(t . \epsilon_{k}(\omega)\right)-E_{\Omega}\left(e^{i\left\langle t, \gamma_{k}\right\rangle}\right) E_{\Omega}\left(\widehat{\zeta}\left(t . \epsilon_{k}\right)\right)\right] e^{i\left\langle t, n_{k}\right\rangle} .
$$

Theorem 4.1. Let $\left\{\gamma_{k}\right\}_{k \in \mathbb{N}^{r}} \subset\left(\mathbb{R}^{+}\right)^{d}$ and $\left\{\epsilon_{k}\right\}_{k \in \mathbb{N}^{r}} \subset(0,1]^{d}$ be two independent sequences of positive i.i.d. random vectors, and $\left\{n_{k}\right\}_{k \in \mathbb{N}^{r}} \subset \mathbb{R}^{+}$. Assume that

(a) $E_{\Omega}\left(\left(\min _{1 \leq j \leq d}\left|\epsilon_{e, j}\right|\right)^{-\alpha}\right)<\infty$, for some $\alpha>0$ and some $e \in \mathbb{N}^{r}$;

(b) $\sum_{k \in \mathbb{N}^{r}} P\left(\left|\gamma_{k}\right|>c 2^{|k|^{r \beta}}\right)<\infty$ for some constants $c \geq 1$, and $0<\beta<1$; and

(c) $\left|n_{k}\right|=O\left(2^{|k|^{r \beta}}\right)$. Let $\zeta: \mathbb{R}^{d} \rightarrow \mathbb{R}$ be positive, integrable with unit integral, with support on $\{|t| \leq 1\}$, and satisfying

(d) $\sup _{t} \prod_{j=1}^{d} \max \left(1,\left|t_{j}\right|\right)^{\alpha}|\widehat{\zeta}(t)|<\infty$.

If $\left\{A_{n}\right\}_{n \in \mathbb{N}}$ is variationally good in $L^{2}$ then $\left\{F_{n}\right\}_{n \in \mathbb{N}}$ is uniformly variationally good in $L^{2}$.

Proof. Fix $e \in \mathbb{N}^{r}$. Since both $\left\{\epsilon_{k}\right\}$ and $\left\{\gamma_{k}\right\}$ are i.i.d. and independent of each other,

$$
E_{n} f=E_{\Omega}\left(F_{n}^{\omega} f\right)=\int_{\left(\mathbb{R}^{+}\right)^{d}} E_{\Omega}\left(\zeta_{\epsilon_{e}}(t)\right) T_{t} A_{n}\left(E_{\Omega}\left(T_{\gamma_{e}} f\right)\right) d t .
$$

Since $\left\{A_{n}\right\}_{n \in \mathbb{N}}$ is variationally good in $L^{2}$, there exists a constant $c(s)>0$ such that

$$
\|\| E_{n} f\left\|_{v(s)}\right\|_{2} \leq \int_{\left(\mathbb{R}^{+}\right)^{d}} E_{\Omega}\left(\zeta_{\epsilon_{e}}(t)\right)\|\| A_{n}\left(T_{\gamma_{e}+t} f\right)\left\|_{v(s)}\right\|_{2} d t \leq c(s)\|f\|_{2} .
$$

Next, we consider the centered averages $\left\{D_{n}^{\omega} f=F_{n}^{\omega} f-E_{n} f\right\}$ along a well chosen subsequence. For $h \geq 1$, let $\left\{a_{j}\right\}_{j \in I_{h}}$ to be the sequence of equally spaced integers such that $2^{h} \leq a_{j}<2^{h+1}, a_{j+1}-a_{j} \sim 2^{h} / h^{2}$, and the smallest element in this 
sequence is $2^{h}$. Rename this sequence $\left\{m_{l}\right\}=\cup_{k} \cup_{j \in I_{h}} a_{j}$.

$$
\begin{aligned}
\|\| D_{m_{l}}^{\omega} f\left\|_{v(s)}\right\|_{2}^{2} \leq & \|\| D_{m_{l}}^{\omega} f\left\|_{v(2)}\right\|_{2}^{2} \leq 4 \sum_{l \geq 1}\left\|D_{m_{l}}^{\omega} f\right\|_{2}^{2} \\
\leq & 4 \sum_{h \geq 1} \sum_{j \in I_{h}} \int_{|t| \leq 2^{2^{r \beta h}}}\left|\widehat{D}_{a_{j}}^{\omega}(t)\right|^{2} d \mu_{f} \\
& +4 \sum_{h \geq 1} \sum_{j \in I_{h}} \int_{|t|>2^{2^{r \beta h}}}\left|\widehat{D}_{a_{j}}^{\omega}(t)\right|^{2} d \mu_{f}=\mathrm{I}+\mathrm{II} .
\end{aligned}
$$

To estimate the first term $I$ we need first to check the requirements of Proposition 2.4. For $A \subset \mathbb{R}^{d}$ (Borel measurable) let $\zeta_{\epsilon_{k}(\omega)}(A)=\int_{A} \zeta_{\epsilon_{k}(\omega)}(t) d t$. Note that since the support of $\zeta \subset\{|t| \leq 1\}$, and $\left|\epsilon_{k}\right| \leq 1$, the support of the measures $\zeta_{\epsilon_{k}(\omega)} \subset\{|t| \leq 1\}$. Let $\nu_{k}(\omega, A)=\delta_{n_{k}+\gamma_{k}(\omega)} * \zeta_{\epsilon_{k}(\omega)}(A)=\zeta_{\epsilon_{k}(\omega)}\left(A-n_{k}-\gamma_{k}(\omega)\right)$. Setting $B=\sup _{k} n_{k} 2^{-|k|^{r \beta}}$, if $\left|\gamma_{k}\right|<c 2^{|k|^{r \beta}},\left[|t|>(B+2 c) 2^{|k|^{r \beta}}\right]-n_{k}-\gamma_{k} \subset$ $\left[|t|>c 2^{|k|^{r \beta}}\right]$. Thus, for large enough $|k|$,

$$
\begin{aligned}
E_{\Omega} \nu_{k}\left(|t|>(B+2 c) 2^{|k|^{r \beta}}\right) \leq & E_{\Omega}\left(\zeta_{\epsilon_{k}}\left(|t|>c 2^{|k|^{r \beta}}\right)\right) \\
& +P\left(\left|\gamma_{k}\right|>c 2^{|k|^{r \beta}}\right)=P\left(\left|\gamma_{k}\right|>c 2^{|k|^{r \beta}}\right) .
\end{aligned}
$$

Now, by Proposition 2.4, assumption (b) and following the same ideas in Theorem 3.3, there exists $0<C \in L^{\infty}(\Omega)$, such that, for $2^{h} \leq a_{j}<2^{h+1}$,

$$
\max _{|t| \leq 2^{2^{\beta h}}}\left|\widehat{D}_{a_{j}}^{\omega}(t)\right|^{2} \lesssim C(\omega) \frac{\log \left(2^{2^{r \beta h}}\right)}{2^{r h}} \lesssim C(\omega) \frac{1}{2^{r h(1-\beta)}} .
$$

Since $\left|I_{h}\right| \sim h^{2}$ and $0<\beta<1$,

$$
\mathrm{I} \lesssim C(\omega) \sum_{h=1}^{\infty} h^{2} \frac{1}{2^{r h(1-\beta)}} \int_{|t| \leq 2^{2^{r \beta h}}} d \mu_{f}=C(\omega) c(\beta)\|f\|_{2}^{2} .
$$

For the second term, the assumptions on $\zeta$ imply

$$
\left|\widehat{D}_{n}^{\omega}(t)\right| \lesssim \frac{1}{|t|^{\alpha}}\left[\frac{1}{n^{r}} \sum_{l \in[1, n]^{r}} \frac{1}{\min _{1 \leq j \leq d}\left|\epsilon_{l, j}(\omega)\right|^{\alpha}}+E\left(\frac{1}{\min _{1 \leq j \leq d}\left|\epsilon_{l, j}\right|^{\alpha}}\right)\right] .
$$

By assumption (a), there exist $0<C_{2} \in L^{1}(\Omega)$ such that $\sup _{n}\left|\widehat{D}_{n}^{\omega}(t)\right| \leq \frac{C_{2}(\omega)}{|t|^{\alpha}}$, thus

$$
\begin{aligned}
\mathrm{II}=\sum_{h} \sum_{j \in I_{h}} \int_{|t|>2^{2^{r \beta h}}}\left|\widehat{D}_{a_{j}}^{\omega}(t)\right|^{2} d \mu_{f} & \leq C_{2}^{2}(\omega) \sum_{h} \frac{h^{2}}{2^{\alpha 2^{r \beta h}}} \int_{|t|>2^{2^{r \beta h}}} d \mu_{f} \\
& \leq C_{2}^{2}(\omega) c(\beta)\|f\|_{2}^{2} .
\end{aligned}
$$

Now let $\tilde{D}_{n}^{\omega}=D_{m_{l}}^{\omega}$ if $m_{l} \leq n<m_{l+1}$. Then $\left\|D_{n}^{\omega} f\right\|_{v(s)}=\left\|D_{n}^{\omega} f-\tilde{D}_{n}^{\omega} f\right\|_{v(s)}+$ $\left\|\tilde{D}_{n}^{\omega} f\right\|_{v(s)}$. The second term is the variation along the subsequence $\left\{m_{l}\right\}$. For the first term, by Proposition 3.5 .

$$
\begin{aligned}
\left\|D_{n}^{\omega} f-\tilde{D}_{n}^{\omega} f\right\|_{v(s)} & \leq 2\left(\sum_{l}\left\|\left\{D_{n}^{\omega} f: m_{l} \leq n<m_{l+1}\right\}\right\|_{v(s)}^{s}\right)^{1 / s} \\
& \leq 2\left(\sum_{l}\left\|\left\{D_{n}^{\omega} f: m_{l} \leq n<m_{l+1}\right\}\right\|_{v(1)}^{2}\right)^{1 / 2}
\end{aligned}
$$


and for $m_{l} \in I_{h}$,

$$
\begin{aligned}
\|\left\{D_{n}^{\omega} f:\right. & \left.m_{l} \leq n<m_{l+1}\right\} \|_{v(1)} \leq \sum_{n=m_{l}}^{m_{l+1}-1}\left|D_{n}^{\omega} f-D_{n+1}^{\omega} f\right| \\
& \leq\left(m_{l+1}-m_{l}\right)^{1 / 2}\left(\sum_{n=m_{l}}^{m_{l+1}-1}\left|D_{n}^{\omega} f-D_{n+1}^{\omega} f\right|^{2}\right)^{1 / 2} \\
& \leq\left(\frac{2^{h}}{k^{2}}\right)^{1 / 2}\left(\sum_{n=m_{l}}^{m_{l+1}-1}\left|D_{n}^{\omega} f-D_{n+1}^{\omega} f\right|^{2}\right)^{1 / 2} .
\end{aligned}
$$

Since $\widehat{D}_{n}^{\omega}$ are bounded, $\left|\widehat{D}_{n}^{\omega}(t)-\widehat{D}_{n+1}^{\omega}(t)\right| \sim \frac{C}{n}$, thus

$$
\begin{aligned}
& \|\| D_{n}^{\omega} f-\tilde{D}_{n}^{\omega} f\left\|_{v(s)}\right\|_{2}^{2} \leq 4 \sum_{h} \frac{2^{h}}{h^{2}} \sum_{j \in I_{h}} \sum_{n=a_{j}}^{a_{j+1}-1}\left\|D_{n}^{\omega} f-D_{n+1}^{\omega} f\right\|_{2}^{2} \\
& \leq 4 \sum_{h} \frac{2^{h}}{h^{2}} \sum_{n=2^{h}}^{2^{h+1}-1}\left\|D_{n}^{\omega} f-D_{n+1}^{\omega} f\right\|_{2}^{2} \\
& \leq 4 \sum_{h} \frac{2^{h}}{h^{2}} \sum_{n=2^{h}}^{2^{h+1}-1} \int\left|\widehat{D}_{n}^{\omega}(t)-\widehat{D}_{n+1}^{\omega}(t)\right|^{2} d \mu_{f} \sim \sum_{h} \frac{2^{h}}{h^{2}} \sum_{n=2^{h}}^{2^{h+1}-1} \frac{1}{n^{2}} \int d \mu_{f} \\
& \sim \sum_{h} \frac{1}{h^{2}}\|f\|_{2}^{2}=C\|f\|_{2}^{2} .
\end{aligned}
$$

Combining the variational inequalities for $\left\{E_{n} f\right\}$ and for the centered averages $\left\{F_{n}^{\omega} f-E_{n} f\right\}$, the theorem is proven.

The key element in the above theorem, is the control of the decay of the averaging kernels in condition (d). Thus, Theorem 4.1 is a particular case of item (c) in the following more general result.

Proposition 4.2. Let $\left\{\nu_{k}\right\}_{k \in \mathbb{N}^{r}}$ be a sequence of transition measures satisfying the assumptions of Theorem 3.3. Let $K_{n}, E_{n}$ and $D_{n}$ as defined in Section 3. If there exist $\alpha>0$ such that $\sup _{n} \sup _{|t| \geq 1}|t|^{\alpha}\left|\widehat{D}_{n}^{\omega}(t)\right| \in L^{1}(\Omega)$, then there exists $0<C \in L^{\infty}(\Omega)$ such that, for any $\rho>1$,

$$
\left\|\sqrt{\sum_{n=1}^{\infty}\left|D_{\rho^{n}} f\right|^{2}}\right\|_{2}<C(\omega) c(\rho)\|f\|_{2} .
$$

Moreover,

(a) If for some $\rho>1,\left\{E_{n}\right\}_{n \in I(\rho)}$ is variationally good in $L^{2}$ then $\left\{K_{n}\right\}_{n \in I(\rho)}$ is uniformly variationally good in $L^{2}$.

(b) If $\left\{E_{n}\right\}$ is universally good in $L^{2}, \lim _{\rho \mapsto 1} \sup _{n} b_{\rho^{n+1}} / b_{\rho^{n}}=1$ and either $\left\{\nu_{k}\right\}$ is a sequence of real-valued positive transition measures or $\left\{\left|E_{n}\right|\right\}$ is also universally good in $L^{2}$, then $\left\{K_{n}\right\}$ is uniformly universally good in $L^{2}$.

(c) Assume the total variation $\left|\nu_{k}(\omega)\right|=1$ for all $k \in \mathbb{N}^{r}$ and all $\omega \in \Omega$, then if $\left\{E_{n}\right\}$ is variationally good in $L^{2}$ then $\left\{K_{n}\right\}$ is uniformly variationally good in $L^{2}$. 
Proof. Proving the $L^{2}$ bound for the square function follows the same ideas as in the proof of the square function in Theorem 3.3 together with the estimate for the second term in Theorem 4.1. Parts (a) and (b) follow as in Theorem 3.3 and (c) follows the same estimates as in the proof of Theorem 4.1

Bounded transition measures with the conditions in Proposition 4.2 also are uniform norm summable. Such result requires Moricz's [29] estimate for moments of sums of random variables and its extension in Cohen and Cuny [16].

Proposition 4.3 ([16]). Let $(Y, \mathcal{C}, \mu)$ a probability space and $\left\{G_{n}\right\} \subset L^{2}(Y)$. Let $\left\{\alpha_{n}\right\}$ a sequence of non-negative numbers, $\eta>0, C>0$ constants, and $\left\{H_{n}\right\}$ a non-decreasing sequence with growth condition $H_{n} \lesssim n^{\eta}$ such that, for all $m>n \geq$ 1 ,

$$
\left\|\sum_{k=n+1}^{m} G_{k}\right\|_{2}^{2} \leq H_{m} \sum_{k=n+1}^{m} \alpha_{k}
$$

If $\sum_{n \geq 1} \alpha_{n} H_{n}(\log n)^{2}<\infty$, then $\sum_{n \geq 1} G_{n}$ converges a.e. in $L^{2}(Y)$, and

$$
\left\|\sup _{n \geq 1}\left|\sum_{k=1}^{n} G_{k}\right|\right\|_{2}^{2} \leq C(\eta) \sum_{n \geq 1} \alpha_{n} H_{n}(\log n)^{2} .
$$

Theorem 4.4. Given a sequence of bounded transition measures $\left\{\nu_{k}\right\}_{k \in \mathbb{N}^{r}}$ such that, for some $\beta \in(0,1)$,

$$
\sum_{k \in \mathbb{N}^{r}} E_{\Omega}\left|\nu_{k}\right|\left(|t|>c 2^{|k|^{r \beta}}\right)<\infty \text { and } M(\omega)=\sup _{n} \sup _{|t| \geq 1}|t|^{\alpha}\left|\widehat{\nu}_{n}^{\omega}(t)\right| \in L^{\infty}(\Omega)
$$

for some $\alpha>0$. Then $\left\{\nu_{k}\right\}$ is uniformly norm summable in $L^{2}$.

Proof. Let $I_{n, m}=\left\{k \in \mathbb{N}^{r}: n<|k| \leq m\right\}$.

$$
\left\|\sum_{k \in I_{n, m}} \frac{\left.\nu_{k} f(x)-E_{\Omega} \nu_{k} f(x)\right)}{|k|^{r}}\right\|_{2}^{2} \leq \int_{\mathbb{R}^{d}}\left|\sum_{k \in I_{n, m}} \frac{\widehat{\nu}_{k}(t)-\widehat{E_{\Omega} \nu_{k}}(t)}{|k|^{r}}\right|^{2} d \mu_{f} .
$$

By Proposition 2.4 and the tail distribution condition on the $\nu_{k}$ 's, there exists $0<C_{1}(\omega) \in L^{\infty}(\Omega)$ such that, for all $|t| \leq 2^{m^{r \beta}}$,

$\left|\sum_{k \in I_{n, m}} \frac{\left.\widehat{\nu}_{k}(t)-{\widehat{E_{\Omega}}}_{k}(t)\right)}{|k|^{r}}\right|^{2}<C_{1}(\omega) \sum_{j=n+1}^{m} \frac{1}{j^{r+1}} \log \left(2^{m^{r \beta}}\right) \sim C_{1}(\omega) m^{r \beta}\left(\sum_{j=n+1}^{m} \frac{1}{j^{r+1}}\right)$.

On the other hand, for $|t|>2^{m^{r \beta}}$,

$$
\left|\sum_{k \in I_{n, m}} \frac{\widehat{\nu}_{k}(t)-\widehat{E_{\Omega} \nu_{k}}(t)}{|k|^{r}}\right|^{2} \lesssim \frac{M(\omega)}{|t|^{2 \alpha}}\left[\sum_{k \in I_{n, m}} \frac{1}{|k|^{r}}\right]^{2} \leq M(\omega) \frac{(\log m)^{2}}{2^{2 \alpha m^{r \beta}}} .
$$

Combining both estimates, there exists $0<C(\omega) \in L^{\infty}(\Omega)$ such that

$$
\left\|\sum_{k \in I_{n, m}} \frac{\widehat{\nu}_{k}(t)-E_{\Omega}\left(\widehat{\nu}_{k}(t)\right)}{|k|^{r}}\right\|_{2}^{2} \lesssim C(\omega) m^{r \beta}\left(\sum_{j=n+1}^{m} \frac{1}{j^{r+1}}\right)\|f\|_{2}^{2} .
$$

Since $\beta<1$, the result follows by Proposition 4.3 . 
Proof of Theorem 1.12, It follows from Theorem 4.1 and Theorem 4.4, with estimates for the kernels as in Theorem 4.1.

\section{ACKNOWLEDGMENT}

We thank the referee for very helpful comments and remarks.

\section{REFERENCES}

[1] Mustafa Akcoglu, Alexandra Bellow, Andrés del Junco, and Roger L. Jones, Divergence of averages obtained by sampling a flow, Proc. Amer. Math. Soc. 118 (1993), no. 2, 499-505, DOI 10.2307/2160329. MR1143221

[2] M. A. Akcoglu, A. del Junco, and W. M. F. Lee, A solution to a problem of A. Bellow, Almost everywhere convergence, II (Evanston, IL, 1989), Academic Press, Boston, MA, 1991, pp. 1-7. MR.1131778

[3] Mustafa Akcoglu, Alexandra Bellow, Roger L. Jones, Viktor Losert, Karin Reinhold-Larsson, and Máté Wierdl, The strong sweeping out property for lacunary sequences, Riemann sums, convolution powers, and related matters, Ergodic Theory Dynam. Systems 16 (1996), no. 2, 207-253, DOI 10.1017/S0143385700008798. MR.1389623

[4] I. Assani, A weighted pointwise ergodic theorem (English, with English and French summaries), Ann. Inst. H. Poincaré Probab. Statist. 34 (1998), no. 1, 139-150, DOI 10.1016/S0246-0203(98)80021-6. MR 1617709

[5] I. Assani, Wiener-Wintner dynamical systems, Ergodic Theory Dynam. Systems 23 (2003), no. 6, 1637-1654, DOI 10.1017/S0143385703000117. MR2032481

[6] I. Assani, Duality and the one-sided ergodic Hilbert transform, Chapel Hill Ergodic Theory Workshops, Contemp. Math., vol. 356, Amer. Math. Soc., Providence, RI, 2004, pp. 81-90, DOI 10.1090/conm/356/06497. MR2087589

[7] V. Bergelson, M. Boshernitzan, and J. Bourgain, Some results on nonlinear recurrence, J. Anal. Math. 62 (1994), 29-46, DOI 10.1007/BF02835947. MR.1269198

[8] Fakhreddine Bouhkari and Michel Weber, Almost sure convergence of weighted series of contractions, Illinois J. Math. 46 (2002), no. 1, 1-21. MR1936072

[9] J. Bourgain, An approach to pointwise ergodic theorems, Geometric aspects of functional analysis (1986/87), Lecture Notes in Math., vol. 1317, Springer, Berlin, 1988, pp. 204-223, DOI 10.1007/BFb0081742. MR950982

[10] Jean Bourgain, On pointwise ergodic theorems for arithmetic sets (English, with French summary), C. R. Acad. Sci. Paris Sér. I Math. 305 (1987), no. 10, 397-402, DOI 10.1007/BF02698838. MR916338

[11] J. Bourgain, Almost sure convergence and bounded entropy, Israel J. Math. 63 (1988), no. 1, 79-97, DOI 10.1007/BF02765022. MR959049

[12] J. Bourgain, H. Furstenberg, I. Katznelson, and D. Orstein, Return time sequences of $d y$ namical systems, Publ. Math. Inst. Hautes Etudes Sci., 69 (1989), Issue 1, 42-45.

[13] Donald L. Burkholder, Sharp inequalities for martingales and stochastic integrals, Astérisque 157-158 (1988), 75-94. Colloque Paul Lévy sur les Processus Stochastiques (Palaiseau, 1987). MR.976214

[14] Guy Cohen, On random Fourier-Stieltjes transforms, Ergodic theory and related fields, Contemp. Math., vol. 430, Amer. Math. Soc., Providence, RI, 2007, pp. 73-88, DOI 10.1090/conm/430/08252. MR2331326

[15] Guy Cohen and Christophe Cuny, On random almost periodic series and random ergodic theory, Ergodic Theory Dynam. Systems 26 (2006), no. 3, 683-709, DOI 10.1017/S0143385705000660. MR2237464

[16] Guy Cohen and Christophe Cuny, On random almost periodic trigonometric polynomials and applications to ergodic theory, Ann. Probab. 34 (2006), no. 1, 39-79, DOI 10.1214/009117905000000459. MR2206342

[17] Guy Cohen, Roger L. Jones, and Michael Lin, On strong laws of large numbers with rates, Chapel Hill Ergodic Theory Workshops, Contemp. Math., vol. 356, Amer. Math. Soc., Providence, RI, 2004, pp. 101-126, DOI 10.1090/conm/356/06499. MR2087591 
[18] Guy Cohen and Michael Lin, Extensions of the Menchoff-Rademacher theorem with applications to ergodic theory: Probability in mathematics, Israel J. Math. 148 (2005), 41-86, DOI 10.1007/BF02775432. MR2191224

[19] Jack Cuzick and Tze Leung Lai, On random Fourier series, Trans. Amer. Math. Soc. 261 (1980), no. 1, 53-80, DOI 10.2307/1998317. MR.576863

[20] Edward Brian Davies, One-parameter semigroups, London Mathematical Society Monographs, vol. 15, Academic Press, Inc. [Harcourt Brace Jovanovich, Publishers], London-New York, 1980. MR591851

[21] Ciprian Demeter, Pointwise convergence of the ergodic bilinear Hilbert transform, Illinois J. Math. 51 (2007), no. 4, 1123-1158. MR2417419

[22] Sylvain Durand and Dominique Schneider, Théorèmes ergodiques aléatoires et suites de poids aléatoires régularisants (French, with English and French summaries), C. R. Math. Acad. Sci. Paris 334 (2002), no. 5, 375-378, DOI 10.1016/S1631-073X(02)02279-3. MR1892937

[23] Aihua Fan and Dominique Schneider, Sur une inégalité de Littlewood-Salem (French, with English and French summaries), Ann. Inst. H. Poincaré Probab. Statist. 39 (2003), no. 2, 193-216, DOI 10.1016/S0246-0203(02)00014-6. MR1962133

[24] Roger L. Jones, Robert Kaufman, Joseph M. Rosenblatt, and Máté Wierdl, Oscillation in ergodic theory, Ergodic Theory Dynam. Systems 18 (1998), no. 4, 889-935, DOI 10.1017/S0143385798108349. MR1645330

[25] Roger L. Jones, Andreas Seeger, and James Wright, Strong variational and jump inequalities in harmonic analysis, Trans. Amer. Math. Soc. 360 (2008), no. 12, 6711-6742, DOI 10.1090/S0002-9947-08-04538-8. MR2434308

[26] Jean-Pierre Kahane, Some random series of functions, 2nd ed., Cambridge Studies in Advanced Mathematics, vol. 5, Cambridge University Press, Cambridge, 1985. MR.833073

[27] B. Krause, Polynomial Ergodic Averages Converge Rapidly: Variations on a Theorem of Bourgain, arXiv:1402.1803 2014.

[28] A. Zygmund, Józef Marcinkiewicz (Polish), Wiadom. Mat. (2) 4 (1960), 11-41 (1960). (1 plate). MR 115885

[29] F. Móricz, Moment inequalities and the strong laws of large numbers, Z. Wahrscheinlichkeitstheorie und Verw. Gebiete 35 (1976), no. 4, 299-314, DOI 10.1007/BF00532956. MR407950

[30] R. Paley and A. Zygmund, On some series of functions, I; II; III. Proc. Cambridge Philos. Soc., 26 (1930), 337-357; 26 (1930), 458-474; 28 (1932), 190-205.

[31] Stephen Parrott, Unitary dilations for commuting contractions, Pacific J. Math. 34 (1970), 481-490. MR268710

[32] Karin Reinhold, A smoother ergodic average, Illinois J. Math. 44 (2000), no. 4, 843-859. MR.1804316

[33] Yao-Feng Ren and Han-Ying Liang, On the best constant in Marcinkiewicz-Zygmund inequality, Statist. Probab. Lett. 53 (2001), no. 3, 227-233, DOI 10.1016/S0167-7152(01)00015-3. MR.1841623

[34] Frigyes Riesz and Béla Sz.-Nagy, Functional analysis, Dover Books on Advanced Mathematics, Dover Publications, Inc., New York, 1990. Translated from the second French edition by Leo F. Boron; Reprint of the 1955 original. MR:1068530

[35] Joseph M. Rosenblatt and Máté Wierdl, Pointwise ergodic theorems via harmonic analysis, Ergodic theory and its connections with harmonic analysis (Alexandria, 1993), London Math. Soc. Lecture Note Ser., vol. 205, Cambridge Univ. Press, Cambridge, 1995, pp. 3-151, DOI 10.1017/CBO9780511574818.002. MR1325697

[36] Walter Rudin, Fourier analysis on groups, Wiley Classics Library, John Wiley \& Sons, Inc., New York, 1990. Reprint of the 1962 original; A Wiley-Interscience Publication. MR 1038803

[37] R. Salem and A. Zygmund, Some properties of trigonometric series whose terms have random signs, Acta Math. 91 (1954), 245-301, DOI 10.1007/BF02393433. MR65679

[38] Dominique Schneider, Convergence presque sûre de moyennes ergodiques perturbées (French, with English and French summaries), C. R. Acad. Sci. Paris Sér. I Math. 319 (1994), no. 11, 1201-1206. MR 1309101

[39] Dominique Schneider, Théorèmes ergodiques perturbés (French, with English summary), Israel J. Math. 101 (1997), 157-178, DOI 10.1007/BF02760927. MR.1484874

[40] Béla Sz.-Nagy and Ciprian Foiaş, Harmonic analysis of operators on Hilbert space, NorthHolland Publishing Co., Amsterdam-London; American Elsevier Publishing Co., Inc., New York; Akadémiai Kiadó, Budapest, 1970. Translated from the French and revised. MR.0275190 
[41] Michel Weber, Estimating random polynomials by means of metric entropy methods, Math. Inequal. Appl. 3 (2000), no. 3, 443-457, DOI 10.7153/mia-03-44. MR1768824

[42] Máté Wierdl, Pointwise ergodic theorem along the prime numbers, Israel J. Math. 64 (1988), no. 3, 315-336 (1989), DOI 10.1007/BF02882425. MR995574

[43] Pavel Zorin-Kranich, Variation estimates for averages along primes and polynomials, J. Funct. Anal. 268 (2015), no. 1, 210-238, DOI 10.1016/j.jfa.2014.10.018. MR.3280058

Division of Mathematics and Computer Science (DMACS), College of Natural and Applied Sciences (CNAS), University of Guam (UOG), AlS 319, UOG Station, Mangilao, 96923 GuAM

Email address: choij@triton.uog.edu

Department of Mathematics and Statistics, University at Albany, SUny, Albany, NEW YORK 12222

Email address: reinhold@albany.edu 